\title{
TRANSPIRATION COOLING USING POROUS MATERIAL FOR HYPERSONIC APPLICATIONS
}

\author{
Adriano Cerminara, Ralf Deiterding and Neil D.Sandham \\ Aerodynamics and Flight Mechanics Research Group, University of Southampton, \\ Southampton, Hampshire, SO17 1 BJ, UK
}

\section{Content}

The present chapter will explore the principal features and main parameters of the mechanism of blowing through a porous surface in a hypersonic flow for wall cooling applications. Results from direct numerical simulations are presented for different configurations, including blowing through discrete slots and through a layer of regular distributed porosity, and discussed with emphasis on their use for the validation of simplified theoretical models. Practical applications for hypersonic flight include the assessment of the influence of the main parameters regulating the flow within the porous medium on the surface cooling performance, as well as on the transition mechanism and the transition location. 


\section{Introduction}

\subsection{Hypersonic environment}

Hypersonic flight is characterized by very high values of temperature and heat flux reached at the wall, which can compromise the structural integrity of the vehicle. This is due to the large amount of kinetic energy converted into thermal energy by the shock forming in front of the body and by viscous effects inside the boundary layer. This produces high temperature peaks inside the boundary layer at small normal distances from the wall, which results in a high wall heat flux. Aerodynamic heating due to viscous effects inside the boundary layer is the main source of wall heating over most of the surface of slender bodies (e.g. cruise vehicles) flying at hypersonic speeds. The leading-edge region near the stagnation point is, in contrast, linked to the high temperatures reached at the edge of the boundary layer due to the front shock. This is the primary form of heating in the case of blunt bodies, such as the re-entry capsules. For a re-entry vehicle flying at a Mach number of 36, for example, as in the case of the Apollo re-entry capsule, the temperature in the shock layer in front of the nose can reach values as high as $11000 \mathrm{~K}$ (Anderson Jr, 2006).

\subsection{Boundary-layer thermal characteristics}

In order to understand the heat-exchange mechanism between the flow and the wall and to predict the temperature levels and heat load to which the vehicle surface is exposed, it is necessary to consider the main thermal characteristics of a hypersonic boundary layer. 
We need to distinguish between two separate regions of the body, namely the stagnation-point region and the surface downstream of the leading edge. If we consider the case of an isothermal wall with a temperature $T_{w}$ (e.g. the room temperature in a hypersonic wind tunnel), the wall heat flux in the stagnation region depends on the temperature difference between the maximum value at the boundary-layer edge (linked to the temperature jump across the shock), and the value at the wall. Moreover, the heat flux depends also on the boundary-layer thickness $(\delta)$, which is, in turn, dependent on the shock-layer thickness and hence on the stand-off distance. As the stand-off distance decreases with the nose radius, this results in the heat flux at the stagnation point being dependent on the nose radius $(R)$, and in particular higher for a sharper nose, with the proportionality $q_{w} \propto 1 / \sqrt{R}$ (Anderson Jr., 2006). Hence the leading-edge heat flux is higher for bodies with a sharper nose. In the case of chemical reactions and vibrational nonequilibrium, the temperature changes within the shock layer due to these effects need to be taken into account, as they determine the value of the temperature at the boundary-layer edge.

On the surface of a slender body, in contrast, the aerodynamic heating is caused by the dissipation of a large amount of kinetic energy (related to the high streamwise speed $u$ at the boundary-layer edge) inside the boundary layer due to viscosity. This represents the main mechanism acting on the surface section downstream of the vehicle leading edge. As a consequence of the viscous effects, the temperature increases continually with reducing velocity as the wall is approached, until it reaches a peak at a certain distance from the surface, and then rapidly decreases to the fixed (low) value at the wall $\left(T_{w}\right)$. As the temperature peak is located near the wall, this results in a steep gradient on the vehicle surface, i.e. a high heat flux. This is a peculiar 
feature of high-speed boundary layers, as compared to low-speed (subsonic) cases, which is due to the fact that in the high-speed case the contribution of the kinetic-energy dissipation in the energy balance within the boundary layer is no longer negligible.

The wall heat flux can be expressed by Fourier's law as

$$
q_{w}=-\left.k \frac{\partial T}{\partial y}\right|_{y=0}
$$

By considering the equations for a two-dimensional (2D) compressible laminar boundarylayer along a flat plate, in the case of high-speed flow where the viscous effects cannot be neglected, it is possible to express the wall heat flux as a function of the total temperature of the flow and the tangential velocity gradient at the wall through the relation

$$
q_{w}=\left.\frac{k\left(T_{w}-T_{0 \infty}\right)}{U_{\infty}}\left(\frac{\partial u}{\partial y}\right)\right|_{y=0}
$$

Equation 2 shows that the wall heat flux depends on the difference between the surface temperature and the fluid total temperature. The total temperature can be obtained, for an isentropic flow, by the relation 


$$
T_{0 \infty}=T_{\infty}\left(1+\frac{\gamma-1}{2} M^{2}\right)
$$

which shows the dependence on the square of the Mach number; thus it assumes very high values (typically on the order of thousands of Kelvin degrees) for flows at high Mach numbers. This means that, in a hypothetical case in which no mechanisms of heat dissipation are present in the material exposed to a hypersonic flow, the surface temperature would increase starting from its initial value $T_{w}$ up to the value of the fluid total temperature. This is of course not sustainable by the material forming the vehicle surface in a real case, and would melt the surface, resulting in failure of the vehicle structure. Also, it should be mentioned that the maximum temperature that the wall would reach in a hypothetical case, and hence the temperature at which the adiabatic condition is reached (i.e. $q_{w}=0$ ), is not exactly the fluid total temperature, but a value slightly lower, which is called adiabatic wall temperature $\left(T_{a w}\right)$, and is defined as

$$
T_{a w}=T_{\infty}\left(1+\frac{\gamma-1}{2} r M^{2}\right)
$$

In the above relation, $r$ is the recovery factor, which is $r=\operatorname{Pr}^{1 / 2}$ for laminar flows and $r=\operatorname{Pr}^{1 / 3}$ in the turbulent case. The latter equation determines the adiabatic wall temperature to be lower than the total temperature of the fluid due to the Prandtl number being different from 1 in a real case.

Finally, equation 2 also shows that the wall heat flux depends directly on the wall shear stress, thus on the skin friction coefficient, which highlights the dramatic effect that transition to turbulence has, in general, on the surface heat flux in hypersonic flows. 
On the basis of the above discussion, it is clear that aerodynamic heating represents a significant challenge in the design process of a hypersonic vehicle. In order to reduce the wall heating effects, hypersonic vehicles need an appropriate thermal protection system (TPS) capable of storing, or radiating/dissipating outwards, the high heat load and keeping the temperature of the internal structure within tolerable values. In the following section the main forms of surface cooling for hypersonic vehicle are reviewed, and the principal parameters and physical properties that are required for the thermal protective layer on the vehicle surface are presented and discussed.

\section{Requirements of Thermal Protection Systems}

The cooling techniques considered for the thermal protection system of hypersonic vehicles can be divided into two main classes, namely active cooling and passive cooling. The active cooling systems make use of a coolant fluid (either gas or liquid) coming in direct contact with the vehicle surface, either with the internal or the external face, in ordertokeepitstemperature within a tolerable range. A passive cooling system, in contrast, makes use of the heat resistance and insulation capabilities of the material forming the TPS to store and radiate outwards the heat received from the hot boundary layer. For example, for lifting bodies, reusable ceramic-matrix and carbon-carbon composite materials have been shown to be the most suitable materials for the TPS, providing high heat-load storage and thermal resistance capabilities. When the capability of radiating the heat outwards is coupled with the presence of a fluid flowing inside the TPS structure, such a system is defined as a semi-passive cooling system. For example, in the case of a heat pipe, the entering heat is carried by an internal working fluid to other sections of the surface where it is radiated outwards. Ablation is also considered a form of semi-passive 
cooling, and is the technique used in the most extreme hypersonic environments, as in the case of ballistic atmospheric re-entry. Ablative systems are generally made of a polymeric-matrix (e.g. epoxy or phenolic resin) composite material that uses a thermochemical degradation process (pyrolysis) of the polymer to carry out the absorbed heat via blowing of the gaseous products of pyrolysis, which carry out a high enthalpy content. Fillers of a composite material can be of different types (e.g. glass microspheres, phenolic microspheres, silica fibres, nylon fibres, carbon fibres, etc.) and their role, in general, is to provide (dependent on the type) thermal resistance and insulation properties, as well as structural integrity (e.g. abrasion resistance) of the TPS. One of the most advanced ablators designed for extremely high space re-entry speeds is the so called PICA (Phenolic Impregnated Carbon Ablator), which is made of a low-density substrate of carbon fibres (Carbon FiberForm) impregnated with phenolic resin (Tran et al., 1997). This material was used for the TPS of the Stardust capsule, which re-entered the Earth atmosphere at a speed of about $12.8 \mathrm{Km} / \mathrm{s}$ (the highest ever reached for a Earth re-entry) experiencing a peak surface temperature of about $3200 \mathrm{~K}$ at an altitude of $65 \mathrm{~km}$ (Winter and Trumble, 2010).

Besides their capability to 'actively' eject the heat through the ablation mechanism, ablative systems owe their efficiency as TPS also to the high insulation (hence passive cooling) properties of the 'char', i.e. the residual carbonaceous layer formed on the surface of the heat shield as a result of the pyrolysis process. This is a particularly porous layer, whose solid structure is composed exclusively by the 'heaviest' and most stable chemical elements that did not undergo gasification, along with the fillers of the composite material. Hence, the char layer combines both the properties of a low thermal conductivity $(k)$ and a high thermal capacity $\left(c_{p}\right)$, thus behaving as a perfect heat sink/insulator, capable of storing a large quantity of enthalpy content at the surface while not 
allowing the heat to be transferred more deeply into the structure. Although the ablative systems can resist the highest heat flux and temperature values encountered in re-entry flight, their disadvantage is that they are not re-usable, as they consume during the re-entry.

If one considers more moderate conditions, as for example those relative to a lifting re-entry (Space Shuttle-like re-entry) or, in more general, a cruise flight (which ideally could cover a Mach number range from about Mach 5 to Mach 10), re-usability becomes a realistic requirement. In such conditions, the Ultra High Temperature Ceramics (UHTC) have been demonstrated to be among the best candidates for the TPS material, thanks to their thermal properties. UHTC materials have a high resistance to thermal shocks, melting points typically in the range $2000 \mathrm{~K}$ $3000 \mathrm{~K}$, as well as a high thermal conductivity and a high re-radiation capability. They are considered suitable for the leading-edge regions of a hypersonic vehicle, which are exposed to high peaks of the heat flux. Instead of storing the heat as in the case of thermal insulation (which requires a low thermal conductivity along with a high thermal capacity), these materials allow the heat being transferred locally to the surface at a high rate (e.g. at the stagnation point) and to be easily conducted within the surface, so that it can be redistributed over a larger area of the surface where it is re-radiated back into the atmosphere. This allows a significant reduction in the heat load absorbed by the leading edge of the vehicle.

Hence, we can summarize the main characteristics of a TPS for a generic hypersonic vehicle as follows: i) high heat storage capability, i.e. high thermal capacity (specific heat, $c_{p}$ ); ii) low thermal conductivity $(k)$, thus high porosity (low density) of the internal structure; iii) high reradiation capability, i.e. high surface emissivity coefficient $(E)$; iv) an internal mechanism that either convects the heat directly outside (e.g. ablation) or transfers it to different regions of the 
surface where it gets dissipated by another mechanism of inverse heat-transfer (e.g. re-radiation); v) light structure, i.e. low specific weight and limited thickness; vi) durability in operating condition.

Note that among the above characteristics, reusability is not mentioned, as it depends on the system being of ablative or non-ablative type, which, in turn, depends on the flight conditions. An important challenge of the next-generation TPS systems for hypersonic flight will be then to push the limit of the TPS reusability up to the higher Mach number conditions, e.g. for re-entry flight or very high-speed cruise flight. This drives the design of the system towards a solution that combines the main features of passive or semi-passive cooling with a direct form of active cooling aimed to decrease the high external heat flux to more sustainable values. A system based on transpiration cooling has, in general, the potential to fill the gap between reusability and efficiency in very harsh environments.

\subsection{Film cooling and transpiration cooling technique}

The principle of active cooling systems is to make use of a coolant to directly decrease the local temperature and heat flux in the external flow and/or in the internal structure. The film cooling technique (Heufer and Olivier, 2008; Keller and Kloker, 2014, 2016), for example, consists of injecting coolant into the hot boundary layer to create a thin cold film of fluid near the wall, thus modifying the thermal as well as kinematic characteristics of the local boundary layer. This directly reduces the external wall heat flux by reducing the gas temperature. If the coolant is injected through discrete holes on the wall, this is known as effusion cooling. This technique is commonly 
used for turbine blades in the form of injection from single holes (Wittig et al., 1996; Baldauf et al., 1999). However, in this case three-dimensional vortical structures are generated in the flowfield, which can reduce the average cooling effectiveness (Heufer and Olivier, 2008). In contrast, injection through two-dimensional (2D) slots leads to higher cooling effectiveness by providing a spanwise homogeneous flowfield (Keller and Kloker, 2016). This is the form of injection mostly investigated for supersonic flow conditions.

The reduction of the heat flux is actually due to a dual mechanism, i.e. a decrease of the local gas temperature near the wall, and an increase of the boundary-layer thickness as a consequence of the coolant mass injection, which leads to a decrease in the velocity and temperature gradients at the wall (hence of the skin friction and heat flux). Downstream of the opening, a laminar boundary layerwill be formed, in general, by two separate layers with different profiles, i.e. an upper region of hot fluid and a bottom layer of cold fluid. The streamwise extent of this separated flow region should be as long as possible to maintain the benefits of cooling. However, after a certain distance the two fluid layers mix together untila hot boundary-layer profile is reestablished. Thus, the holes should be adequately distributed over the surface and the coolant blowing ratio $\left(F=\rho_{c} v_{c} / \rho_{\infty} u_{\infty}\right)$ should be controlled to provide a target cooling efficiency. The performance of a film-cooling system is quantified by the cooling effectiveness, which is defined as (Heufer and Olivier, 2006)

$$
\eta=\frac{T_{a w}-T_{a w, c}}{T_{a w}-T_{0, c}} .
$$


In the above equation, the term $T_{a w, c}$ is the adiabatic wall temperature in the presence of cooling, and $T_{0, c}$ is the total temperature of the coolant gas, which is equal to the temperature inside the plenum chamber. The cooling effectiveness in this form requires that the system is given sufficient time to shift from a steady state of maximum temperature equal to the adiabatic wall temperature of the hot gas, to another steady state in which the maximum wall temperature reached (corresponding to zero heat flux) is lower due to the effect of cooling. In a wind-tunnel experiment, the test time is usually too small to allow for the adiabatic conditions to be reached. The wall temperature can be considered, in general, as fixed and equal to the room temperature. The plenum temperature can be assumed equal to the room temperature as well.

In the transpiration cooling technique, instead, cooling is achieved by a cold fluid transpiring from a porous material. The cold fluid traversing the thickness of the porous material reduces the temperature of the interior porous walls and brings the absorbed heat content outside (a form of reverse heat flux similar to an ablative system). In this case, the coolant injection into the boundary layer is not localized, but distributed over the surface, and irregular due to the interior structure of the porous material. For the flow through porous media at very low Reynolds numbers (lower than 1), the flow rate per unit mass and area coming out from the surface (named also specific discharge or superficial velocity) is linked to the pressure gradient within the porous layer, the fluid viscosity, and the permeability of the material from the Darcy law:

$$
v=-\frac{K_{D}}{\mu} \frac{\partial p}{\partial x}
$$

where $K_{D}$ is the permeability coefficient (the Darcy coefficient in this case), and $x$ is taken as the 
direction of the traversing flow within the material.

For higher Reynolds numbers, however, inertial effects are important and need to be added to the driving force related to the pressure gradient. Hence, the equation with the terms rearranged to express the pressure gradient, takes the form of the Darcy-Forchheimer equation (Langener et al., 2011; Ifti et al., 2018),

$$
-\frac{\partial p}{\partial x}=\frac{\mu}{K_{D}} v+\frac{\rho}{K_{F}} v^{2}
$$

in which $K_{F}$ is the Forchheimer permeability coefficient. Hence, in a transpiration cooling system the material permeability plays a fundamental role for the blowing ratio and the cooling effectiveness.

The permeability coefficient $K_{D}$ can, in turn, be expressed as a function of two other important parameters, namely the porosity $(\varphi)$ and the hydraulic tortuosity $(T)$, as givenin (Matyka et al., 2008)

$$
K_{D}=c_{0} \frac{\phi^{3}}{T^{2} S^{2}}
$$

where $S$ is the specific surface area,i.e. the ratio of the interstitial surface area of the pores to the bulk volume, and $c_{0}$ a constant (Kozeny constant) which depends on the internal geometry. The tortuosity is defined as the ratio between the average path length $(\lambda)$ of the fluid going from the bottom surface to the upper surface of the porous layer and the thickness of the layer $(l)$, 


$$
T=\frac{\lambda}{l}
$$

The capabilities of a transpiration cooling system can then be assessed and optimized once the parameters defining the material permeability, namely the porosity and tortuosity, are known. However, the above-mentioned models for the permeability and tortuosity are not universal, and may differ significantly with the flow conditions and the material, in general. Also, inhomogeneities of the internal material structure will affect the flow traversing the porous layer, leading to non-negligible differences in the blowing ratio as well as the thermodynamic properties of the coolant gas on the surface. Hence, direct numerical simulations capable of resolving the smaller scales within the porous structure can provide physical insights into the flow through a porous medium in hypersonic flow conditions, which can have important consequences on the efficiency of the thermal protection system.

\section{Numerical Method}

\subsection{Governing equations}

We consider numerical solutions of the three-dimensional Navier-Stokes equations for compressible flows, written in conservation form, under the assumption of a perfect gas. The set of non-dimensional conservation equations in Cartesian coordinates can be written as 


$$
\begin{gathered}
\frac{\partial \rho}{\partial t}+\frac{\partial \rho u_{j}}{\partial x_{j}}=0, \\
\frac{\partial \rho u_{i}}{\partial t}+\frac{\partial \rho u_{i} u_{j}}{\partial x_{j}}=-\frac{\partial p}{\partial x_{i}}+\frac{1}{R e} \frac{\partial \tau_{i j}}{\partial x_{j}}, \\
\frac{\partial \rho E}{\partial t}+\frac{\partial \rho E u_{j}}{\partial x_{j}}=-\frac{1}{(\gamma-1) \operatorname{RePr} M^{2}} \frac{\partial}{\partial x_{j}}\left(\mu \frac{\partial T}{\partial x_{j}}\right)-\frac{\partial p u_{j}}{\partial x_{j}}+\frac{1}{R e} \frac{\partial \tau_{i j} u_{i}}{\partial x_{j}}
\end{gathered}
$$

The terms $\rho, \rho u, \rho v, \rho w$ and $\rho E$ are the conservative variables of the system of equations, where $\rho$ is the density, $u, v$ and $w$ are the velocity components respectively in the $x, y$ and $z$ directions, and $E$ is the total energy per unit mass. In the flux vectors, the terms $p, T, \tau_{i j}$, and $\mu$ are respectively the pressure, the temperature, the components of the viscous stress tensor, and the dynamic viscosity of the flow. The non-dimensional quantities are obtained through normalization of the dimensional variables with their freestream reference values, namely $\rho_{\infty}^{*}, U_{\infty}^{*}$ (freestream main velocity), $U_{\infty}^{*}{ }_{\infty}^{2}$ (energy per unit mass and volume), $\rho_{\infty}^{*} U_{\infty}^{*}{ }_{\infty}^{2}$ (term linked to the dynamic pressure, used to normalize pressure and shear stress components), $T_{\infty}^{*}$ and $\mu^{*}{ }_{\infty}$. Note that the superscript ( ${ }^{*}$ ) is used to denote dimensional values. The characteristic length chosen to normalize the length scales is the boundary-layer displacement thickness $\left(\delta^{*}\right)$. Time scales are normalized with respect to the fluid dynamic characteristic time $\left(\delta^{*} / U_{\infty}^{*}\right)$, based on the velocity of the undisturbed flow and on the characteristic length. The terms $\operatorname{Re}, \operatorname{Pr}, M$, and $\gamma$ are respectively the Reynolds, Prandtl and Mach numbers, and the ratio of specific heats $\left(\gamma=c_{p}{ }^{*} / c_{v}{ }^{*}\right)$, i.e. the dimensionless parameters of the flow. The Reynolds number is defined with respect to the boundary-layer displacement thickness of the similarity solution, as $R e=\left(\rho_{\infty}{ }^{*} U_{\infty}{ }^{*} \delta^{*}\right) / \mu_{\infty}{ }^{*}$; the Prandtl number is set to 0.72 for air, and $\gamma$ is equal to 1.4 , as we are considering a perfect gas 
model. The dynamic viscosity is, in turn, expressed in terms of temperature by Sutherland's law

$$
\mu=T^{3 / 2} \frac{1+C}{T+C}
$$

where the constant $C$ represents the ratio between the Sutherland's constant (set to $110.4 \mathrm{~K}$ ) and the reference temperature in the freestream $T_{\infty}^{*}$. The viscous stresses are defined in terms of the velocity derivatives, under the assumption of a Newtonian fluid, as

$$
\tau_{i j}=\mu\left(\frac{\partial u_{i}}{\partial x_{j}}+\frac{\partial u_{j}}{\partial x_{i}}-\frac{2}{3} \delta_{i j} \frac{\partial u_{k}}{\partial x_{k}}\right)
$$

We also need a relation linking the total energy to the temperature, which in non-dimensional form can be expressed as

$$
E=\frac{T}{\gamma(\gamma-1) M^{2}}+\frac{1}{2}\left(u^{2}+v^{2}+w^{2}\right) .
$$

Finally, the system of equations is closed by the equation of state for a perfect gas:

$$
p=\frac{1}{\gamma M^{2}} \rho T
$$




\subsection{Code features}

The numerical simulations have been carried out using the AMROC software framework (Deiterding, 2005a,b, 2011) (Adaptive Mesh Refinement in Object-oriented C++), which uses a hybrid WENO-centred-difference (WENO-CD) scheme in conjunction with a structured adaptive mesh refinement (SAMR) approach. The implementation relative to the base central scheme is equipped with the option to be tuned (or optimized) for spectral resolution improvement, which denotes the scheme as WENO-tuned-centred-difference (WENO- TCD), as described by Hill and Pullin (2004). The shock-capturing filter, namely the WENO scheme, corresponds to the type of WENO-symmetric-order-optimized (WENO-SYMOO) scheme as shown by Martim et al. (2006). The schemes are integrated with a switching function that turns on/off the WENO method at discontinuities/smooth regions.

The base scheme (central scheme) has been proven to have a maximum formal order of accuracy of 6, corresponding to a 7-point stencil, with relative stencil coefficients as in Ziegler et al. (2011). The order of accuracy can be properly specified, which changes the stencil, and can be also reduced (keeping constant the stencil) for optimization of the bandwidth resolution capabilities. For example, considering the 7-point stencil, if the optimization option is specified, the corresponding scheme will be a 4-th order accurate scheme with optimized bandwidth properties (Hill and Pullin, 2004), thus has a minimum dispersion error of the modified wavenumber, suitable for LES (Large-Eddy-Simulation) simulations. Details of the WENO-CD scheme can be found in Cerminara et al. (2018b). The code has been tested to 6-th order accuracy (for both CD and the WENO parts) for both the 2D and the 3D porous wall test cases, giving very good results (which will be shown later in this paper), thus showing to be suitable for DNS computations of the 
current problem.

Finally, the code works in conjunction with a structured adaptive mesh refinement (SAMR) algorithm (Deiterding, 2005a, 2011; Pantano et al., 2007), through which the uniform grid is dynamically refined with consecutive finer grid levels during the iteration cycles, following a patchwise refinement strategy. This applies in regions of the flow where numerical instabilities, related to low initial grid resolution, are detected by means of error indicators.

\section{Results from DNS in hypersonic flow over porous surfaces}

In this section we will be showing numerical results of simulations resolving a hypersonic flow over porous surfaces, with and without the injection of cold fluid. Two porous surface configurations have been considered, namely a simpler case of slots, which is representative of a case of film cooling by effusion, and a case of a regular porous structure, with distributed solid spherical elements, which mimics arealistic porous material, as in the case of transpiration cooling. The flowfield characteristics in both cases are presented, as well as numerical aspects linked to the resolution of the porous structure, and the cooling performance is discussed in relation to the effects on transition.

\subsection{Flat plate with periodic regular pores}

Thefirst configuration is a streamwise and spanwise periodic flat plate with square-shapedholes. The simulations have been run for both a $2 \mathrm{D}$ and a 3D configuration, thus in the 2D configuration the pores reduce to slots. Figure 1 shows the geometrical configurations considered in the $2 \mathrm{D}$ (left) and 3D (right) simulations. The Mach number is 6, the Reynolds number based on the initial boundary layerdisplacement thickness is 6000 , the freestream temperature is $T_{\infty}=216.65 \mathrm{~K}$, and the 
wall temperature is fixed to the adiabatic wall temperature $T_{w}=1522.4 \mathrm{~K}$.

The reference length is the displacement thickness of the initial laminar boundary layer, determined from a similarity solution, through which the flowfield is initialized in our numerical simulations. The simple 2D geometry has been chosen for validation test cases, consisting of 2 pores with regular shape in the $x$-direction. The size of the computational domain is 3 times the initial boundary-layer displacement thickness in the $x$-direction, and 11 times in the $y$-direction. The pores have an $x$-wise length of 0.75 , and a depth of 1 . The large extension of the domain in the $y$-direction is due to the need to allocate enough vertical space for the propagation of disturbance waves generated by the pores.

\subsubsection{Code verification and grid study}

Preliminary 2D simulations have been carried out for the Reynolds number $R e=6000$ to validate the code and test its performances in comparison to SBLI, a DNS code developed over a number of years at the University of Southampton, which has been extensively tested and validated in the past, for example by De Tullio et al. (2013).

Figure 2 shows solutions of the pressure field at the non-dimensional time $t=10$ obtained with the codes AMROC and SBLI, respectively. The mesh resolutions out of the pores for the simulations run with AMROC and SBLI are $64 \times 400$ and $64 \times 356$ (in the $x$ - and $y$-directions) respectively, whereas inside the pores 40 cells are used in the $y$-direction for the AMROC case and 131 points for the SBLI case. The difference in the number of cells/points in the $y$-direction outside and inside the pores between AMROC and SBLI is due to the fact that the grid used in 
AMROC is uniform in both directions, while the grid used in SBLI is stretched in the $y$ direction to increase the resolution towards the wall. The $y$-spacing in the inner-pore region is kept constant and equal to the spacing at the wall in SBLI.

As can be seen, very similar results are obtained with the two codes, which demonstrates the accuracy capabilities of our considered code (AMROC). Also, the figures show that pressure waves are formed at the pore edges, which propagate outwards influencing the flow outside the boundary layer. It has been verified that the amplitude of the radiated pressure waves in the freestream, normalized with the freestream mean pressure, is about a factor of 1.4 higher than the amplitude of the associated normalized density waves, which characterizes acoustic disturbances.

Figure 3 shows a direct comparison between AMROC and SBLI for the density solution along $x$ at $y=1$. The result confirms the good agreement between the two different codes in accurately resolving the acoustic signal produced by the pores. A grid resolution study has been carried out for the case at $R e=6000$, based on the trend of the density along the streamwise direction at $y=1$ (figure 4) and the pressure along the wall-normal direction at $x=1.5$ (figure 5). Six different grid resolution levels (for the base grid level in the SAMR framework) have been analysed to investigate the effect of the grid refinement in the $x$ - and $y$-directions (both simultaneously and separately), namely: $32 \times 440,64 \times 440,128 \times 440,64 \times 660,128 \times 660$, $128 \times 880$ (indicating the number of cells in the $x$ - and $y$-directions respectively).

Both figures show a very good grid convergence. In particular, the solution for the oscillation peaks in figure 4 is shown to be more sensitive to the grid resolution in the $y$-direction (when shifting from 440 to 660 cells), compared to the refinement in the $x$-direction. Figure 5 shows the pressure solution inside the boundary layer, which proves that at the considered grid resolution, 
i.e. $64 \times 440$, without the need for adding dynamically more refinement levels (through the SAMR methodology), we already manage to resolve the boundary layer very well for the present configuration.

\subsubsection{Effect of wall cooling}

The wall cooling effect is analysed in the $2 \mathrm{D}$ configuration for a Reynolds number $R e=$ 20000 by comparing results for the temperature and pressure fields obtained at time $t=50$ for different wall-temperature conditions, namely i) $T_{w}=T_{a d}$ (i.e. adiabatic wall temperature, with $\left.T_{a d}=7.027 T_{\infty}\right)$, ii) $T_{w}=0.8 T_{a d}$, and iii) $T_{w}=0.5 T_{a d}$. The two latter cases correspond to a wallcooling effect with wall temperature decreased by $20 \%$ and $50 \%$ relative to the adiabatic value respectively.

Figure 6 shows the temperature fields for the three above-mentioned wall temperature conditions, while figure 7 shows the corresponding result for the pressure field. As expected, with reference to figure 6 , wall cooling produces an overall decrease of the temperature inside the boundary layer, which results in a lower boundary-layer thickness at the lower wall temperatures. This effect is related to the increase in density inside the boundary layer as a result of the cooling. The most remarkable effect of wall cooling is observed in the pressure field (shown in figure 7), and in particular on the generated acoustic waves. It is evident that, as we move from the adiabatic wall-temperature case to the coldest case, a decrease of the wall temperature produces a decrease of the pressure inside the pores, which results in a stronger expansion at the left corner of each pore and in a stronger compression at the right corner. The pressure waves radiated by the 
pore edges into the boundary layer and the external field are then stronger in the colder wall cases.

\subsubsection{D results - effect of Reynolds number}

The length of the 3D domain is 3 in both the $x$-and the $z$-directions, while the number of pores along each direction, compared to the $2 \mathrm{D}$ case, has been increased to 4 , thus providing a $3 \mathrm{D}$ porous layer composed overall of 16 equally-spaced square-shaped pores. The pores have a length of 0.375 in both the $x$-and the $z$-directions. This configuration corresponds to the configuration analysed in the reference DNS study of De Tullio and Sandham (2010). High-resolution DNS simulations have been run for the $3 \mathrm{D}$ configuration with a mesh size $128 \times 660 \times 128$, using the $6^{\text {th }}$-order WENO- CD method, for both the Reynolds numbers $(R e=6000$ and $R e=20000)$. The results of these simulations prove the high-resolution DNS capabilities of the considered hybrid method for the present case, and show interesting physical features of a hypersonic flow over a porous coating.

Figure 8 shows a generic 3D view of the vertical velocity field, which gives a first impression of the 3D flowfield and the orientation of the radiated waves outside the boundary layer along the streamwise and spanwise directions. Figure 9 shows a comparison of the pressure fields on a $z y$-slice at $x=1.85$ for the two cases. Here, the colour range has been restricted to a smaller band in order to better notice the differences between the two cases in the outer-pore region. We observe higher-amplitude radiated pressure waves and a higher pressure level within the pores for the lower Reynolds number case. A modulation of the pressure field along the spanwise direction in the immediate outer-pore zone is also evident, which is a result of the $3 \mathrm{D}$ porosity of the wall. A further analysis of the rms (root-mean-square) levels of the pressure disturbances outside the 
boundary layer (conducted at the height $y=1.5$ ) has shown rms levels of the pressure fluctuations (normalized with the freestream pressure of the initial unperturbed flow) of 0.045 and 0.0154 for the lower and the higher Reynolds numbers respectively. Thus, at the lower Reynolds number, the pressure waves radiated by the porous wall are approximately 3 times stronger than the waves radiated in the higher Reynolds number case.

The vertical velocity field in the pore region is analysed both in an $x y$-plane at the spanwise position $z=1.85$ (in figure 10) and in an $x z$-plane at the vertical position $y=0$ (in figure 11), namely at the surface plane. Figure 10 shows that higher absolute values of the inwards (negative) and the outwards (positive) vertical velocity are reached just above the pores at the lower Reynolds number, whereas more pronounced values are obtained for the higher Reynolds number inside the pores. A higher vertical velocity is observed outside of the boundary layer for the lower Reynolds number case, which is consistent with the flow being deflected by the presence of higheramplitude radiated acoustic waves.

In figure 11 the three-dimensionality of the inwards and outwards flow on the $y=0$ surface is observed. Higher absolute values are seen for the lower Reynolds number case, consistent with the above discussion. However, in the lower Reynolds number case the flow features shown in figure 11 appear more two-dimensional than in the higher Reynolds number case. In fact, for the $R e=6000$ case the maximum absolute values of the vertical velocity are localized in spanwise-elongated narrow strips adjacent to the pore left and right edges. At $R e=20000$, in contrast, the maximum of the inwards velocity is detached from the left side, and localized in a region between the pore centre and the right side. This effect in turn produces a higher recirculation in the immediate innerpore region, as seen from the higher internal absolute values shown in figure 10 for the higher 
Reynolds number case.

\subsection{Flat plate domain with injection through localized slots}

We consider in this section a long flat plate domain with fluid injection through localized slots, with inlet and outlet boundary conditions. The present configuration has been considered for the study of the effects of fluid injection at different plenum pressures on transition, as well as on the cooling performance over the 3D surface. The flow conditions considered in the present study are related to ongoing experimental investigations in the field of film cooling in hypersonic flow, and are as follows: Mach number $M=5$, unit Reynolds number $\operatorname{Re}_{m}=12.6 \times 10^{6} \mathrm{1} / \mathrm{m}$, and freestream temperature $T_{\infty}=81.7 \mathrm{~K}$. The computational domain used for the case of a flat plate with slots and a plenum chamber is sketched in figures 12,13 and 14 . The size of the domain is $L_{x} \times L_{y} \times L_{z}=200 \times 32 \times 50$, including the sum of the heights of slots and plenum chamber, equal to 2.5. Thus, the distance of the top boundary from the flat plate surface is 29.5. The slots have a height of 1.5, and the depth of the plenum chamber is 1 . A close-up of the slot and the plenum chamber, along with the relative dimensions in an $x y$-plane, is presented in figure 13 . The initial condition is the laminar boundary layer from a similarity solution. The characteristic length is the boundary-layer displacement thickness of the similarity profile at the inflow boundary, i.e. $\delta^{*}=1$ mm. The Reynolds number based on the displacement thickness is then $R e=12600$. The boundary-layer thickness $\left(u_{\mathrm{e}}=0.99 U_{\infty}\right)$ of the similarity inflow is $\delta^{*}=1.25 \mathrm{~mm}$. The growth of the boundary layer in the streamwise direction for the initial state is then imposed following the method described in De Tullio et al. (2013). All the length scales in the present study are normalized with respect to $\delta^{*}$. The inflow boundary in our numerical investigation corresponds 
a distance of $127 \mathrm{~mm}$ from the plate leading edge. Further details about the settings of the simulations can be found in Cerminara et al. (2018a).

At the inlet, afixed inflow condition is imposed in the supersonic region of the boundary layer and in the uniform freestream, whereas an extrapolation condition is imposed in the subsonic region of the boundary layer. The top boundary and the outlet are treated with a standard outflow boundary condition. A plenum boundary condition is used at the bottom boundary, through which the pressure and temperature are set equal to the corresponding stagnation values $\left(p_{0}, T_{0}\right)$ in the plenum. Note that in the work of Keller and Kloker (2014) the same plenum boundary condition was set at the bottom boundary of each slot, as the presence of the plenum was not simulated. Our simulations, instead, consider the presence of a plenum chamber at the bottom of the slots. Figure 14 shows a top view on the surface of the 3D domain. The grid is uniform in each direction, with a size (for the base level in the AMR framework) of $800 \times 384 \times 100$. Two and three overall grid levels are used in the AMR methodology, dependent on the particularcase, which provides minimum cell sizes in $x, y$ and $z$ of $0.125,0.0415$ and 0.25 , for the two level case, and $0.0625,0.0207$, and 0.125 , for the 3 level case. Simulations have been run for four different values of the plenum pressure, namely $p_{0}=1.2 p_{\infty}, 1.5 p_{\infty}, 2 p_{\infty}$, and $3 p_{\infty}$, which provide gradually higher blowing ratios. Two grid levels have been used for the first three of the above mentioned cases, whereas three levels have been used for the latter (i.e. the case with the highest plenum pressure), to better resolve the turbulent region. A constant plenum temperature of $T_{0}=3.67$, corresponding to a dimensional value of $300 \mathrm{~K}$, is considered for all the configurations and for each case with different plenum pressure. Figure 15 shows results for streamlines coloured with temperature in the injection region at the different plenum pressures. These results provide an 
indication of how film cooling is active just downstream of the slots and the effects on the boundary layer of an increasing plenum pressure. As can be seen, for the lowest plenum pressure case, a very thin laminar layer of cold fluid is formed near the wall downstream of the slots and underneath the upper layer of hotter fluid coming from the upstream boundary layer. This is an ideal situation, as the boundary layer is only slightly disturbed by the coolant injection and transition is not triggered immediately downstream of the slots. Also, the thin coolant film is initially separated from the hot boundary layer (before mixing further downstream), which keeps the high temperatures of the hot boundary layer away from the wall. In the second case of $p_{0}=1.5 p_{\infty}$, the coolant layer just downstream of the slots is still laminar, but thicker due to the higher blowing ratio. In this case the hot boundary layer seems to be completely merged within the layer of cold fluid already at the slot location, and the cold layer is longer than in the previous case. These appear to be all positive aspects, however the higher thickening of the boundary layer induced in the higher plenum pressure case needs to be taken into account in terms of the effect that this can have on transition. The flow separates upstream of the slots, and a small recirculation region is induced, as shown by the high-temperature streamlines on the left side of the figure. If this change in the flow enhances the boundary-layer unstable modes, or generates additional unstable modes, this may cause transition to happen at an earlier location than in the case without cooling. As we increase the plenum pressure the boundary layer gets more and more perturbed, and at the highest plenum pressure considered it appears highly distorted and in a highly vortical state, which is likely to be a prelude to the occurrence of transition a short distance downstream.

Figure 16 shows the boundary layer state downstream of the slots through contours of the streamwise velocity at the height $y=1$ for different plenum pressures. As we can see, at the plenum 
pressure $p_{0}=1.5 p_{\infty}$ the boundary layer is still laminar up to the end of the computational domain, but streamwise-elongated structures are generated, which modify the stability characteristics of the boundary layer further downstream. In particular, two high-velocity streaks are formed at the edges of the slots, which are positioned in between of two weaker low-velocity streaks at the sides and the larger low-velocity wake in the middle. Moreover, we notice the presence in the downstream region of 2D-wavy structures (normal to the streamwise direction) gradually growing along the wake, which interact with the streamwise streaks causing their progressive distortion. At the plenum pressure $p_{0}=2 p_{\infty}$ the high-velocity streaks and the wake appear in a highly disturbed state just downstream of the slots, which causes the streaks to break down further downstream and begin the transition process. At the highest plenum pressure case, a more violent transition process is triggered, with the streaks that appear partially fragmented and interacting with the wake flow already in the early region downstream of the slots. In this case, the breakdown mechanism is much more rapid and leads to a turbulent state downstream of $x=115$. The transition region is observed to spread downstream following a wedge-shaped structure.

Figure 17 shows the cooling effectiveness on the surface at the different plenum pressures. As can be seen, the higher values (close to 1) are localized around the slot region and along the wake. Whereas, values close to zero are reached along the main streamwise streaks and in the turbulent region, assuming locally negative values in high compression regions.

Figure 18 quantifies the cooling performance at different plenum pressures by showing the trend of the surface temperature along the midspan axis. As the wall is at adiabatic conditions, the temperature values represent the maximum temperature reached along the surface in the presence of cooling, which in turn gives an indication of the maximum temperature decrease induced by 
the coolant injection. As can be seen, for each case a strong temperature drop is reached at the slot location, which is then followed by a gradual increase downstream. In the case of plenum pressure $p_{0}=1.5 p_{\infty}$ (laminar case), the temperature profile is smooth up to about $x=100$, then pronounced oscillations, related to the 2D waves observed along the wake in figure 16 (top), develop and grow downstream until reaching a peak at approximately $x=170$. These oscillations may be representative of an unstable boundary-layer mode formed in this region of the flow. For the higher plenum pressure case, $p_{0}=2 p_{\infty}$, the temperature profile presents irregular shortwavelength oscillations due to the transitioning flow, but the overall trend and absolute values are still comparable to those of the lower plenum pressure case over most of the domain length. At the highest plenum pressure case, in contrast, transition is triggered just downstream of the slot region, and the surface temperature shows a pronounced increase downstream of about $x=80$. In the transitional region, from $x=80$ to $x=120$, the oscillations are stronger, with very high local peaks. Then (downstream of $x=120$ ), after the turbulent state is reached, both their wavelength and amplitude reduce, and their average value is approximately equal to that relative to the other two cases. In this case, the cooling benefits are already lost in the early region downstream of the slots due to transition.

\subsection{Injection through a porous layer}

For a correct characterization of a transpiration cooling system it is important to analyse the properties of the injected flow through a porous material. The Darcy-Forchheimer equation (7) relates the pressure gradient across the thickness of the porous layer to the injected flow rate through the fluid viscosity, the inertial forces, and the permeability characteristics of the material. 
As we have seen in Section 3.1, the material permeability, or, in other words, the capability of the material to be traversed by a fluid flow, depends on its porosity, i.e. the empty-to-total volume ratio, and on the tortuosity, which is in general defined as the average path length of the streamlines traversing the porous layer. The latter is hence dependent on the particular arrangement of the internal solid elements, for a given porosity. In the context of DNS simulations, a simplified model of an internal porous structure is represented by spherical particles of a certain radius, distributed within the porosity layer with a certain arrangement. The radius and number of the particles will determine, with respect to the total volume of the layer, the porosity as well as the internal length scale of the pores. In order to compare different tortuosity levels in a systematic way, it is more appropriate to compare different regular distributions of the spherical elements, instead of random ones. Hence, regularly distributed spherical elements can be used to carry out a parametric study of the flow features through a porous layer for different porosities and tortuosities. This can give important indications on the regime of validity of the Darcy-Forchheimer equation, and its modified versions, as well as providing insights in the physics of the flow through a porous medium by resolving the smaller porosity scales through direct numerical simulation.

In this section we present preliminary results obtained considering the above mentioned approach, which show some initial interesting physical aspects, and provide a basis for future detailed studies aimed at the analysis of transpiration cooling systems. An example of a regular arrangement of spherical elements is shown in figure 19, which represents the pressure field within a porous layer. In this case, 2D simulations have been run to carry out an initial grid study and an assessment of the porous structure model. Hence, the solid elements are to be considered as cylinders of an infinite length. The solid elements have been created by using an immersed 
boundary method (Deiterding et al., 2006; Deiterding, 2009). The same Cartesian method used for the other flow regions is then used to mesh the region within the porous layer. As can be seen, the element arrangement follows a face-centred-cubic structure, where the $x y$-plane can be imagined as a face of a cube in a 3D space, with the same plane repeating periodically along the $z$ axis in a 3D case. Such an arrangement can be compared to a body-centred-cubic type in a 3D simulation. In the present case, a porosity of $40 \%$ has been imposed, to mimic the behaviour of realistic material samples currently investigated for transpiration cooling systems.

The computational domain is a local flat-plate domain with imposed periodicity in the $x$ direction. The domain dimensions are $L_{x}=5$ and $L_{y}=10.5$, the overall thickness of the region underneath the surface is 2 , whereas the thickness of the porous layer is 1.156 , including the diameter of the cylindrical elements at the edges. The freestream conditions are the same considered in Section 5.2 for the case of the flat plate with slots. The reference length is $1 \mathrm{~mm}$, which corresponds to the similar boundary-layer displacement thickness of the initial condition. The cylinder elements have a radius of $0.078 \mathrm{~mm}$, and the smallest distance between two adjacent elements is $0.02 \mathrm{~mm}$. Hence, the minimum length scale of the internal porous structure is of the order of $20 \mu \mathrm{m}$ in this case. The internal area defined by the centres of the cylindrical elements at the edges is $1 \times 1 \mathrm{~mm}^{2}$. A plenum boundary condition has been imposed at the bottom boundary, with $T_{0}=300 \mathrm{~K}$ and $p_{0}=1.5 p_{\infty}$. As can be seen, a strong pressure gradient is formed in the lower sections of the porous layer, while the pressure adapts to the freestream value in the upper layer. Results refer to the simulation time $t=20$.

The AMR methodology is very important for resolving the smaller scales within the porous layer. For the present case, a grid study has been carried out to find the minimum grid resolution, 
thus the minimum number of refinement levels, required for resolving the porosity length scales with a negligible influence of the grid size. Three grids have been considered, namely a base grid of $75 \times 189$ with 4 overall levels, a base grid of $50 \times 126$ with 5 levels, and a base grid of $50 \times$ 126 with 6 levels. The corresponding minimum cell sizes in $x$ and $y$ are (in order from the first to the last of the grids listed above) $\Delta x_{\min }=0.0083,0.00625,0.003125$, and $\Delta y_{\min }=0.0069,0.0052$, 0.0026. The grid resolution for the finest grid (with up to 6 levels) is shown in figure 20. The results of the grid study are presented in figure 21 for the blowing ratio along the $x$-direction on the surface $(y=0)$. As observed, the two more refined grids show, in general, a quite good agreement both at the surface and inside the porous layer. A local disagreement between these two grids is observed along the surface at the points where the blowing ratio profile crosses the zero value. At these points, in fact, there is an inflection of the profile, which is more pronounced for the highest grid as a result of a smoother surface of the cylindrical elements. Figure 22 shows the trend of the cooling effectiveness along the $x$-direction on the surface (for the finest grid), which reveals that in this case the cooling effectiveness is negative on the surface of the porous material. This is due to the fact that the imposed plenum pressure is not high enough to provide a sufficiently high blowing ratio to cool the wall of the porous material. In contrast to the case of the slots, the internal solid structure of the porous layer works as an obstacle to the injected flow. As a result, the flow undertakes a strong compression in the lower sections of the porous layer (as seen in figure 19), which causes a further increase of the local temperature, i.e. the temperature of the coolant. If the expansion in the upper sections of the porous layer is not high enough (i.e. the blowing ratio is relatively low), the injected fluid results to be a heated fluid from the internal flow compression, which does not perform a cooling effect, but a reverse effect 
instead. This proves that the thermodynamic effects taking place within the porous layer play a significant role on the cooling performance of transpiration cooling systems in hypersonic flow. Figure 23 shows a close-up of the blowing ratio ( $y$-momentum) in the pore region and at the surface for the highest resolution grid. The figure highlights details of the blowing ratio, in particular the alternative negative and positive zones on the surface, as seen already in figure 21 , and the higher positive values reached in the points of minimum distance between two adjacent cylinders in the inner porous layer, corresponding to the strong pressure gradient region observed in figure 19. Figure 24 shows the streamwise and vertical velocity fields in the whole domain. These plots show that the boundary layer has not been significantly distorted by the blowing through the porous layer, as observed by the approximately constant boundary-layer thickness, but weak acoustic waves have been released outside of the boundary layer, which travel in the freestream at the Mach angle $\left(11.5^{\circ}\right.$ for the present case $)$.

\section{Conclusion}

The in-depth analysis of the main features of the flow through porous media in transpiration cooling systems for use on hypersonic vehicles is still an ongoing activity. The present results already show important physical aspects concerning wall cooling performance and boundary-layer properties over a porous wall with and without injection. Different configurations have been considered and tested, namely a periodic flat-plate domain with square-shaped holes, a long flat plate domain with injection through localized slots, and a local flat-plate domain with injection through a layer of distributed porosity. Flow regimes at Mach 5 and 6 have been investigated, dependent on the configuration. 
Results indicate that the presence of holes on the body surface generates acoustic waves that are radiated into the freestream and propagate at the Mach angle, whose amplitude is dependent on the wall temperature condition, and the Reynolds number. For the slot injection case, perturbation induced by the local injection generates streaks inside the boundary layer, which interact with 2D waves growing along the wake further downstream. At higher plenum pressures, transition occurs just beyond the slots, which is seen to cancel the benefits of wall cooling by promoting an earlier increase of the wall temperature with very high local peaks. Whereas, in the case of injection through a layer of distributed porosity, the obtained blowing ratio, for the same plenum pressure case, is much smaller, and the boundary layer only slightly distorted compared to the case of slot injection, as a consequence of the permeability of the porous layer.

Future three-dimensional simulations including different porosities and different arrangements of the solid particles (i.e. different tortuosities) will assess the validity of the Darcy-Forchheimer equation for modelling the injected flow rate through a porous material at different hypersonic flow conditions, as well as giving further insights in the physics of the injected flow through porous media and its effects on the cooling performance and the transition mechanism in transpiration cooling applications.

\section{Acknowledgements}

The authors would like to acknowledge support from EPSRC (Engineering and Physical Sciences Research Council) under the Grant No. EP/P000878/1. 


\section{References}

Anderson Jr, J. D. (2006). Hypersonic and high-temperature gas dynamics. American Institute of Aeronautics and Astronautics.

Baldauf, S., Schulz, A., and Wittig, S. (1999). High resolution measurements of local heat transfer coefficients by discrete hole film cooling. In ASME 1999International Gas Turbine and Aeroengine Congress and Exhibition, pages V003T01A017- V003T01A017. American Society of Mechanical Engineers.

Cerminara, A., Deiterding, R., and Sandham, N. (2018a). Direct numerical simulation of blowing in a hypersonic boundary layer on a flat plate with slots. In 2018 Fluid Dynamics Conference, page 3713.

Cerminara, A., Deiterding, R., and Sandham, N. (2018b). DNS of hypersonic flow over porous surfaces with a hybrid method. In 2018 AIAA Aerospace Sciences Meeting, page 0600.

De Tullio, N., Paredes, P., Sandham, N., and Theofilis, V. (2013). Laminar-turbulent transition induced by a discrete roughness element in a supersonic boundary layer. Journal of Fluid Mechanics, 735:613-646.

De Tullio, N. and Sandham, N. D. (2010). Direct numerical simulation of breakdown to turbulence in a Mach 6 boundary layer over a porous surface. Physics of Fluids, 22(9):094105.

Deiterding, R. (2005a). Construction and application of an AMR algorithm for distributed memory computers. In Adaptive Mesh Refinement-Theory and Applications, pages 361-372. Springer. 
Deiterding, R. (2005b). Detonation structure simulation with AMROC. In International Conference on High Performance Computing and Communications, pages 916-927. Springer.

Deiterding, R. (2009). A parallel adaptive method for simulating shock-induced combustion with detailed chemical kinetics in complex domains. Computers \& Structures, 87:769-783.

Deiterding, R. (2011). Block-structured adaptive mesh refinement - theory, implementation and application. European Series in Applied and Industrial Mathematics: Proceedings, 34:97150.

Deiterding, R., Radovitzky, R., Mauch, S. P., Noels, L., Cummings, J. C., and Meiron, D. I. (2006). A virtual test facility for the efficient simulation of solid materials under high energy shock-wave loading. Engineering with Computers, 22(3-4):325-347.

Heufer, K. and Olivier, H. (2006). Film cooling for hypersonic flow conditions. In Thermal Protection Systems and Hot Structures, volume 631.

Heufer, K. and Olivier, H. (2008). Experimental and numerical study of cooling gas injection in laminar supersonic flow. AIAA journal, 46(11):2741-2751.

Hill, D. J. and Pullin, D. I. (2004). Hybrid tuned center-difference-WENO method for large eddy simulations in the presence of strong shocks. Journalof Computational Physics, 194(2):435450.

Ifti, H. S., Hermann, T., and McGilvray, M. (2018). Flow characterisation of transpiring porous media for hypersonic vehicles. In 22nd AIAA International Space Planes and Hypersonics 
Systems and Technologies Conference, page 5167.

Keller, M. A. and Kloker, M. J. (2014). Effusion cooling and flow tripping in laminar supersonic boundary-layer flow. AIAA Journal, 53(4):902-919.

Keller, M. A. and Kloker, M. J. (2016). Direct numerical simulation of foreign-gas film cooling in supersonic boundary-layer flow. AIAA Journal, 55(1):99-111.

Langener, T., Wolfersdorf, J. V., and Steelant, J. (2011). Experimental investigations on transpiration cooling for scramjet applications using different coolants. AIAA journal, 49(7):1409-1419.

Lombardini, M., Pullin, D., and Meiron, D. (2014). Turbulent mixing driven by spherical implosions. part 1. Flow description and mixing-layer growth. Journal of Fluid Mechanics, 748:85-112.

Mart'ın, M. P., Taylor, E. M., Wu, M., and Weirs, V. G. (2006). A bandwidth-optimized WENO scheme for the effective direct numerical simulation of compressible turbulence. Journal of Computational Physics, 220(1):270-289.

Matyka, M., Khalili, A., and Koza, Z. (2008). Tortuosity-porosity relation in porous media flow. Physical Review E, 78(2):026306.

Pantano, C., Deiterding, R., Hill, D. J., and Pullin, D. I. (2007). A low-numerical dissipation patch-based adaptive mesh refinement method for large-eddy simulation of compressible flows. J. Comput. Phys., 221(1):63-87. 
Tran, H., Johnson, C., Rasky, D., Hui, F., Hsu, M., Chen, T., Chen, Y., Paragas, D., and Kobayashi, L. (1997). Phenolic impregnated carbon ablators pica as thermal protection system for discovery missions. NASA TM-110440, NASA, Washington, DC.

Winter, M. W. and Trumble, K. A. (2010). Spectroscopic observation of the stardust re-entry in the near UV with slit: Deduction of surface temperatures and plasma radiation.

Wittig, S., Schulz, A., Gritsch, M., and Thole, K. (1996). Transonic film-cooling investigations: effects of hole shapes and orientations. In ASME 1996 International Gas Turbine and Aeroengine Congress and Exhibition, pages V004T09A026-V004T09A026. American Society of Mechanical Engineers.

Ziegler, J. L., Deiterding, R., Shepherd, J. E., and Pullin, D. I. (2011). An adaptive high-order hybrid scheme for compressive, viscous flows with detailed chemistry. Journal of Computational Physics, 230(20):7598-7630. 

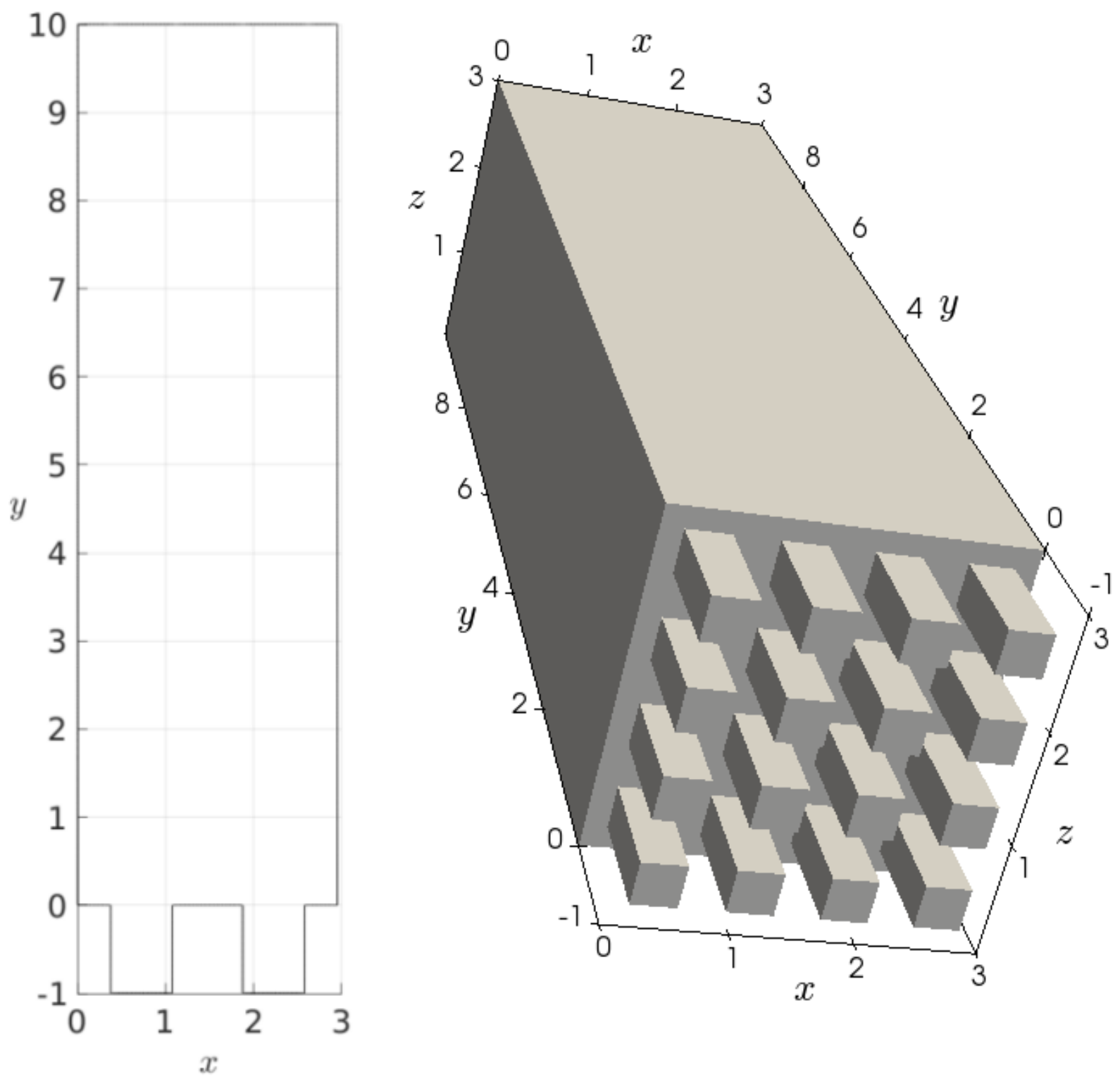

Figure 1: Computational domain geometries for the 2D (left) and 3D (right) simulation 

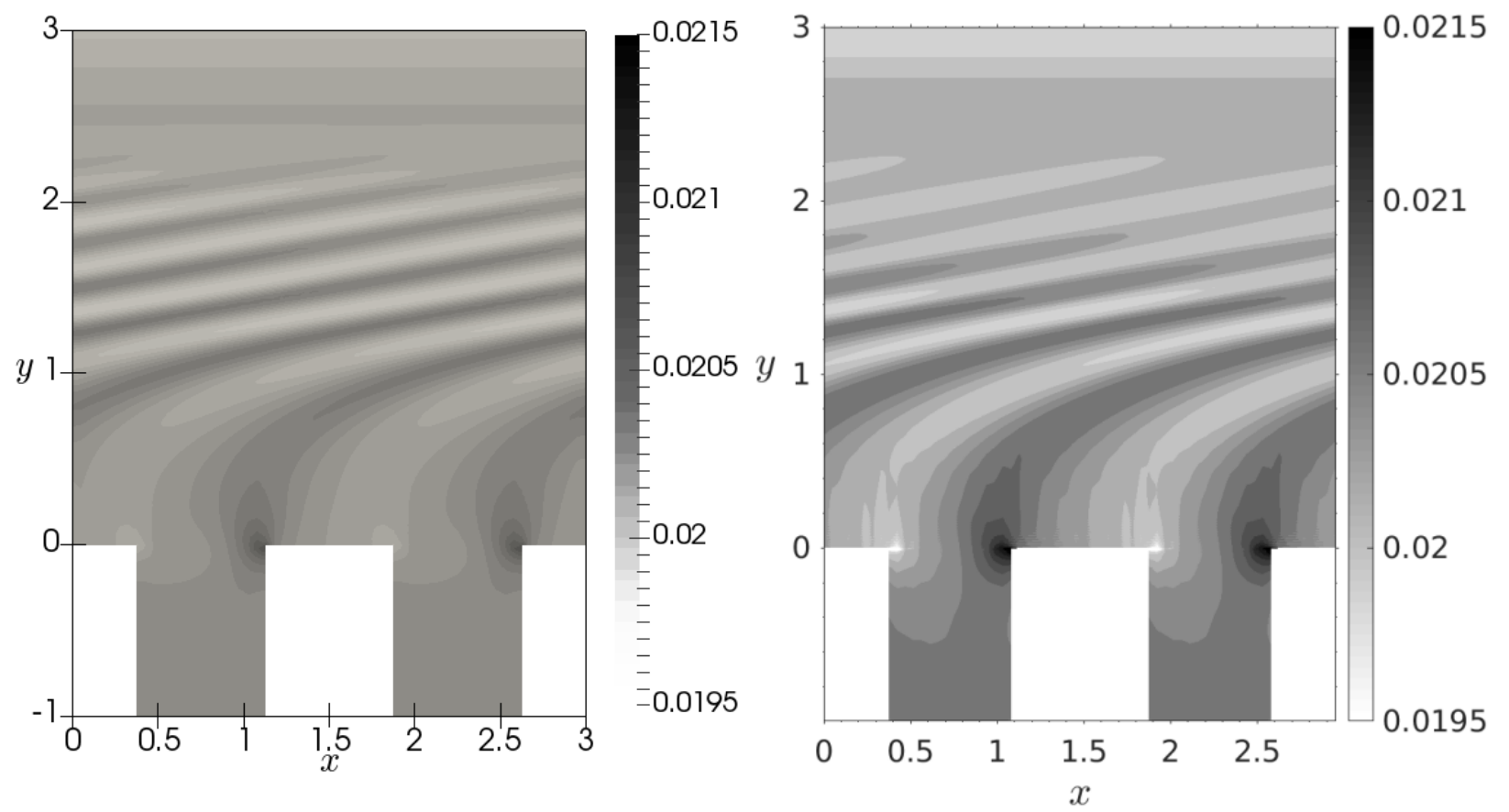

Figure 2: Pressure field at the time $t=10$ obtained with AMROC (left) and SBLI (right)

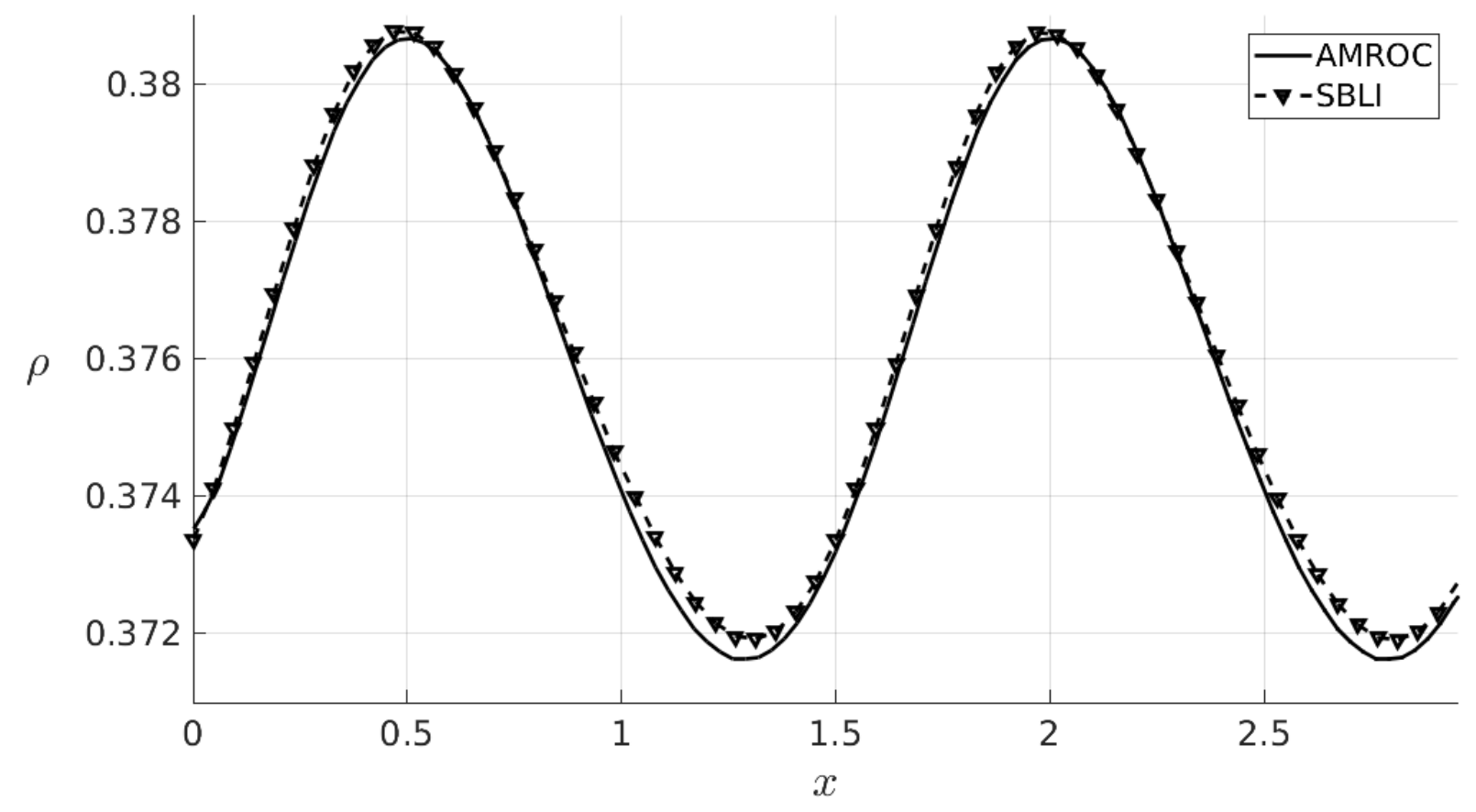

Figure 3: Comparison between AMROC and SBLI for the density trend along $x$ at $y=1$. Time $t=$ 10. 


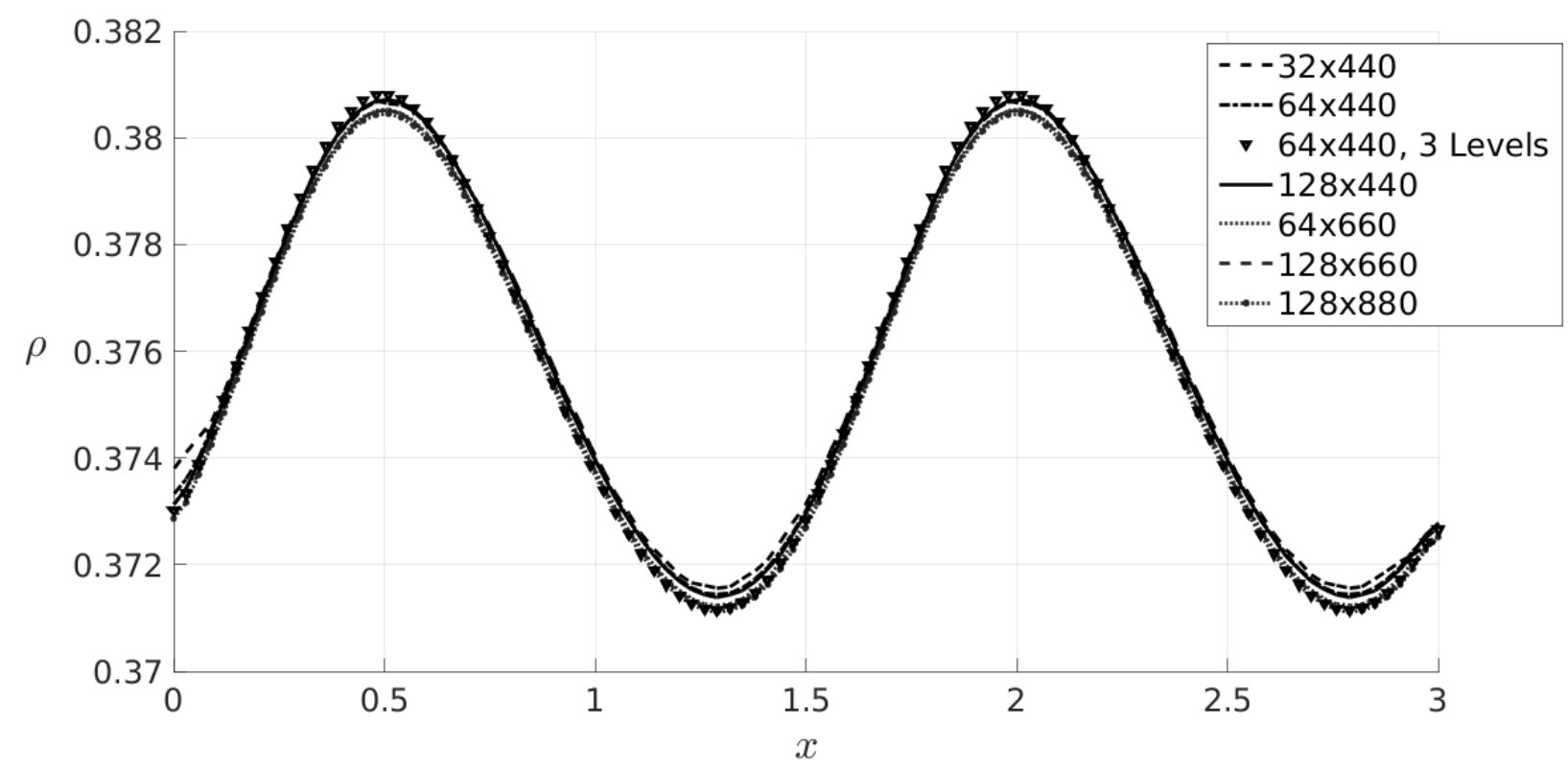

Figure 4: Grid resolution study for the density along $x$ at $y=1, R e=6000$

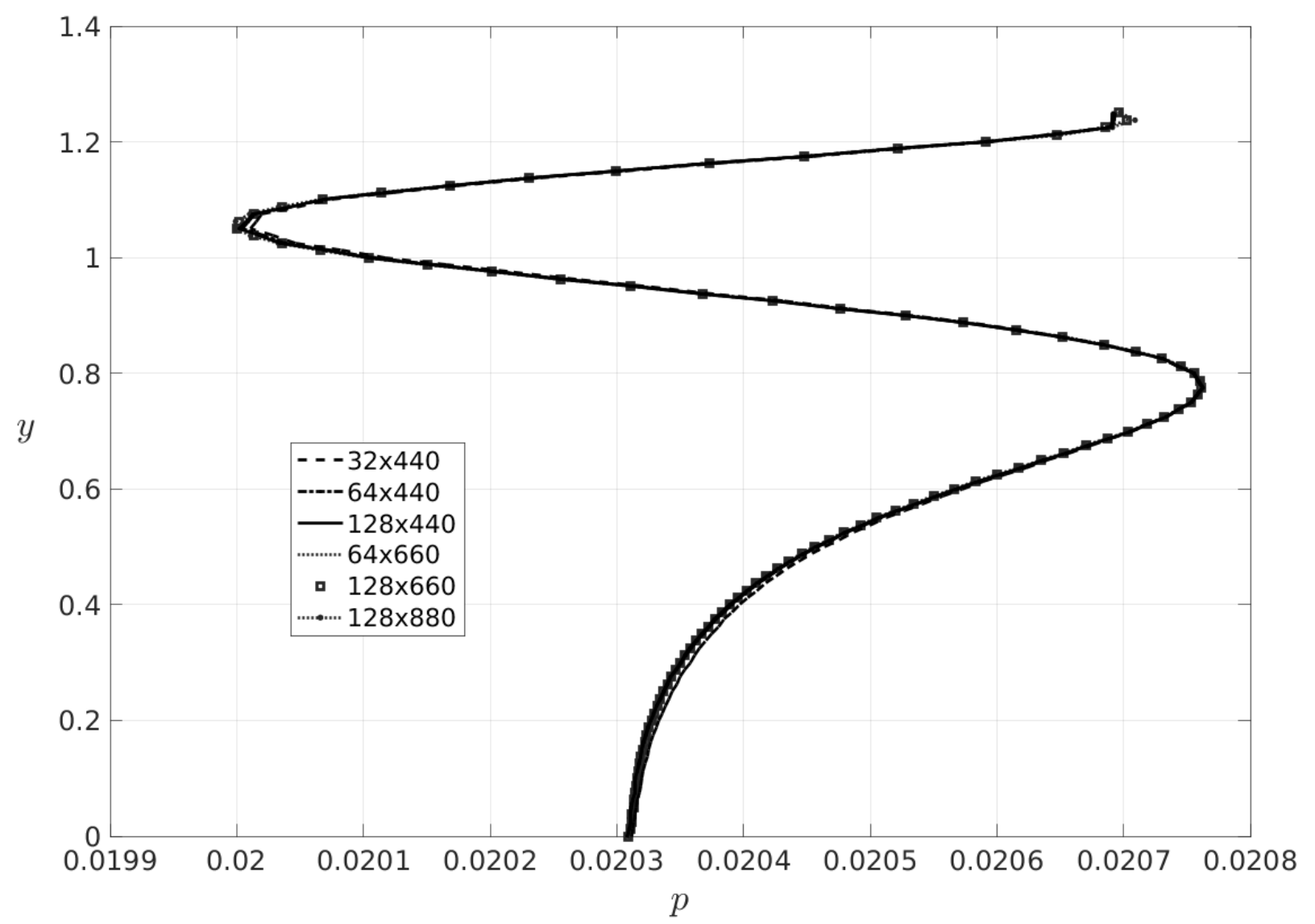

Figure 5: Grid resolution study for the pressure along $y$ at $x=1.5, R e=6000 T$ 

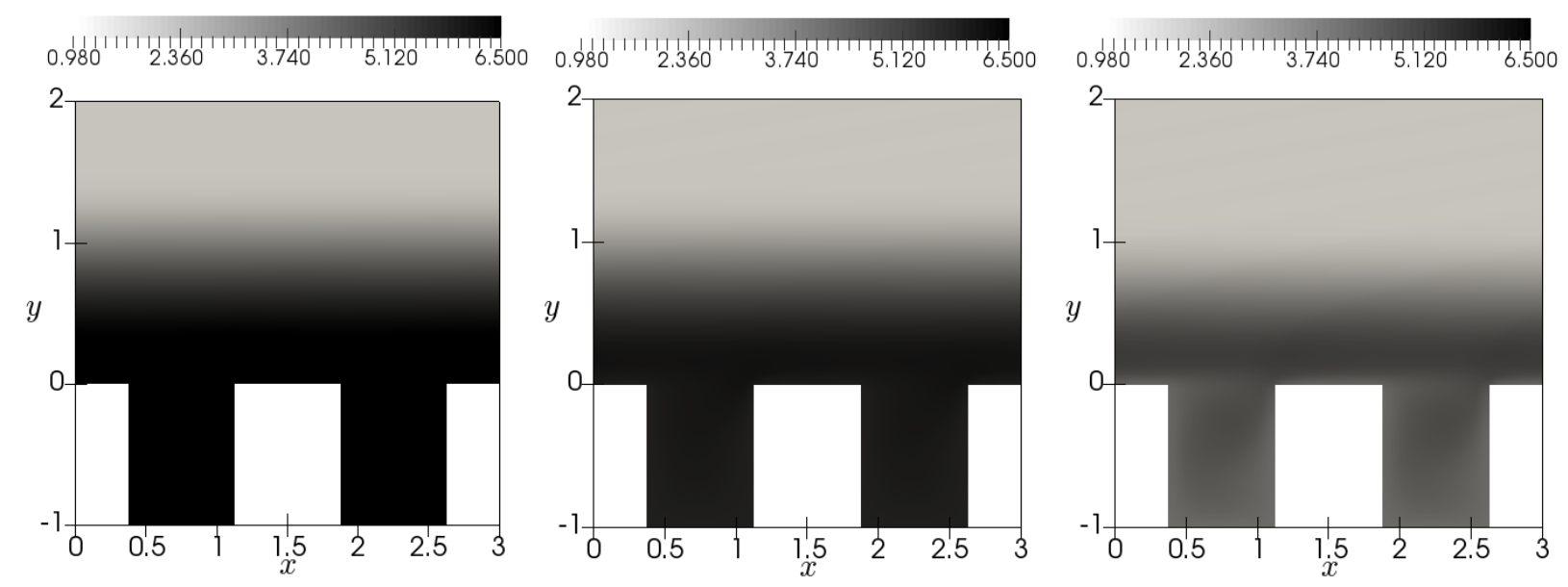

Figure 6: Temperature field in the near-pore region for the wall temperature fixed to the values $T_{w}=$ $T_{a d}$ (left), $T_{w}=0.8 T_{a d}$ (middle) and $T_{w}=0.5 T_{a d}$ (right). Time $t=50, R e=20000$
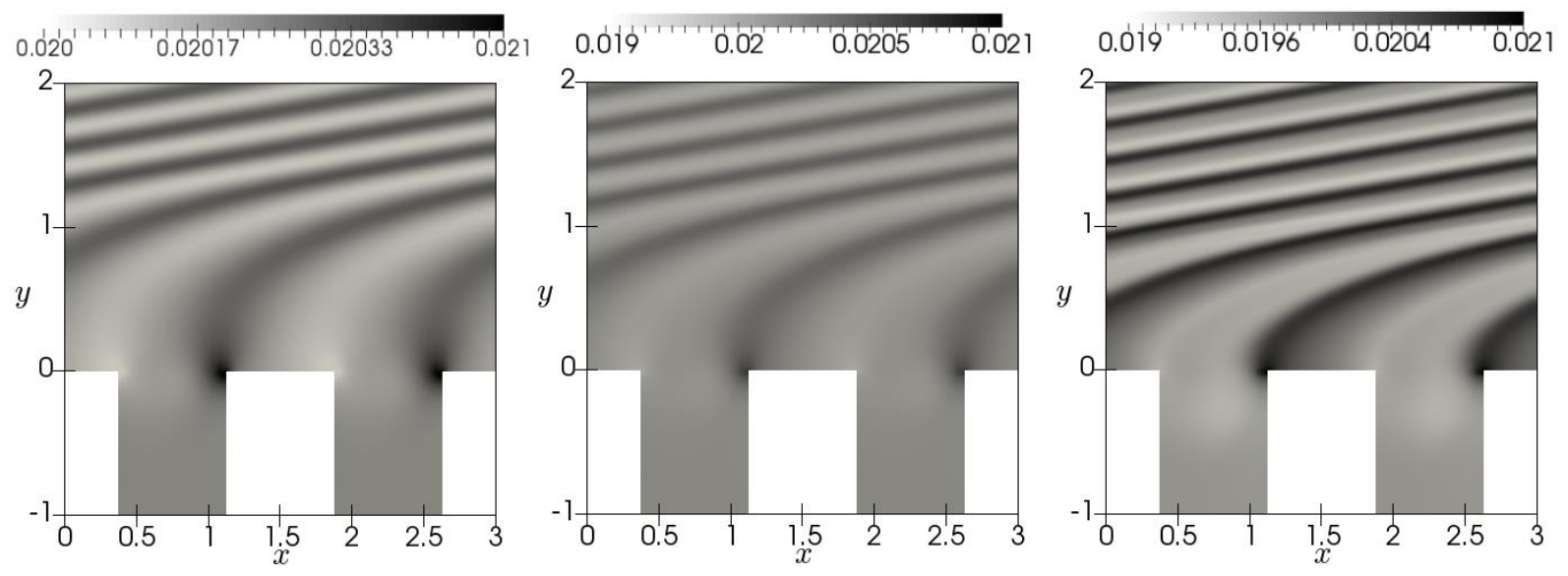

Figure 7: Pressure field in the near-pore region for the wall temperature fixed to the values $T_{w}=$ $T_{a d}$ (left), $T_{w}=0.8 T_{a d}$ (middle) and $T_{w}=0.5 T_{a d}$ (right). Time $t=50, R e=20000$ 


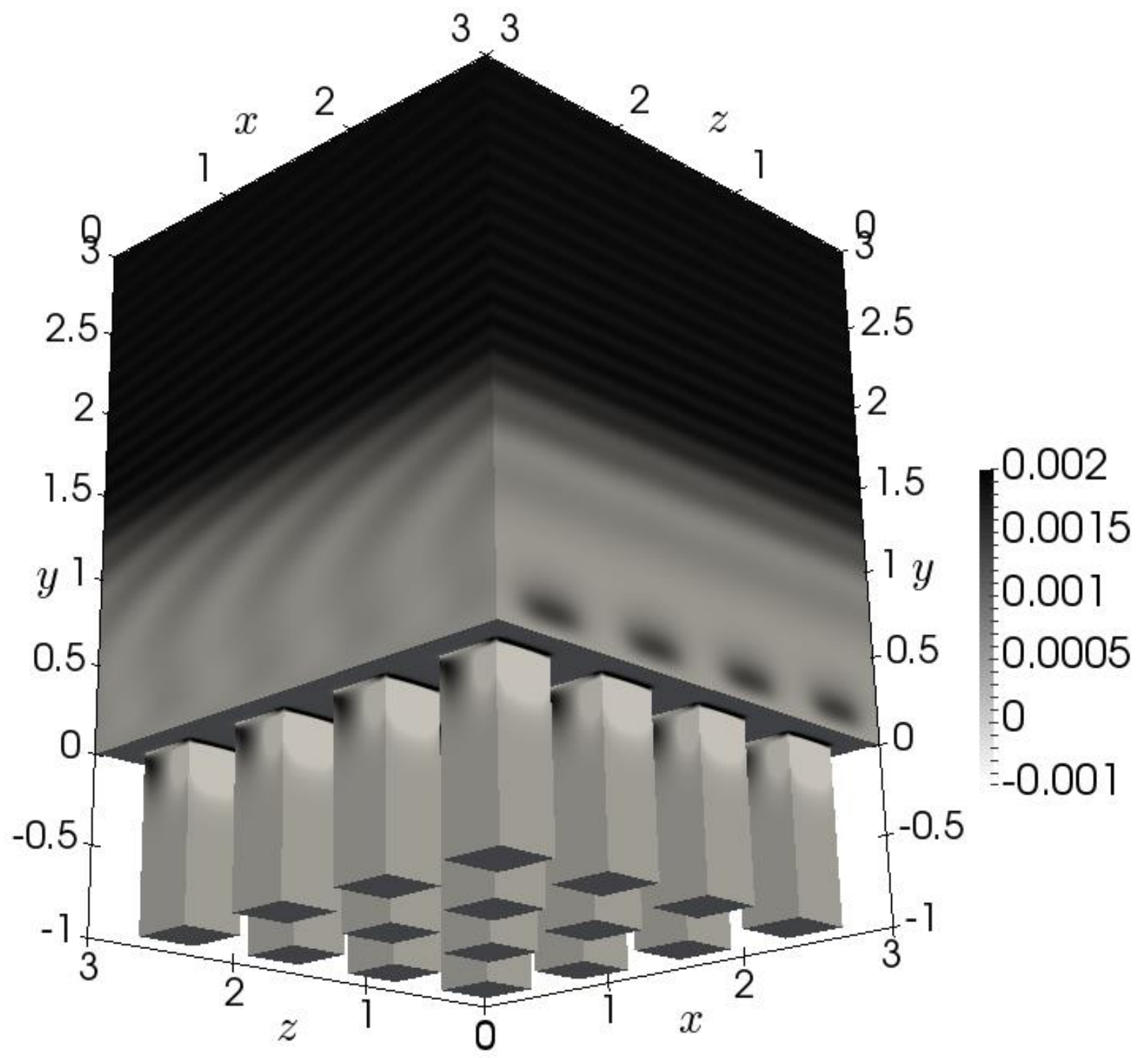

Figure 8: $3 \mathrm{D}$ view of the vertical velocity field. Time $t=50, R e=20000$. Note the inclination of the radiated waves with respect to the $x$-axis outside of the boundary layer, and the spanwise modulation of the velocity field in the immediate outer-pore region 

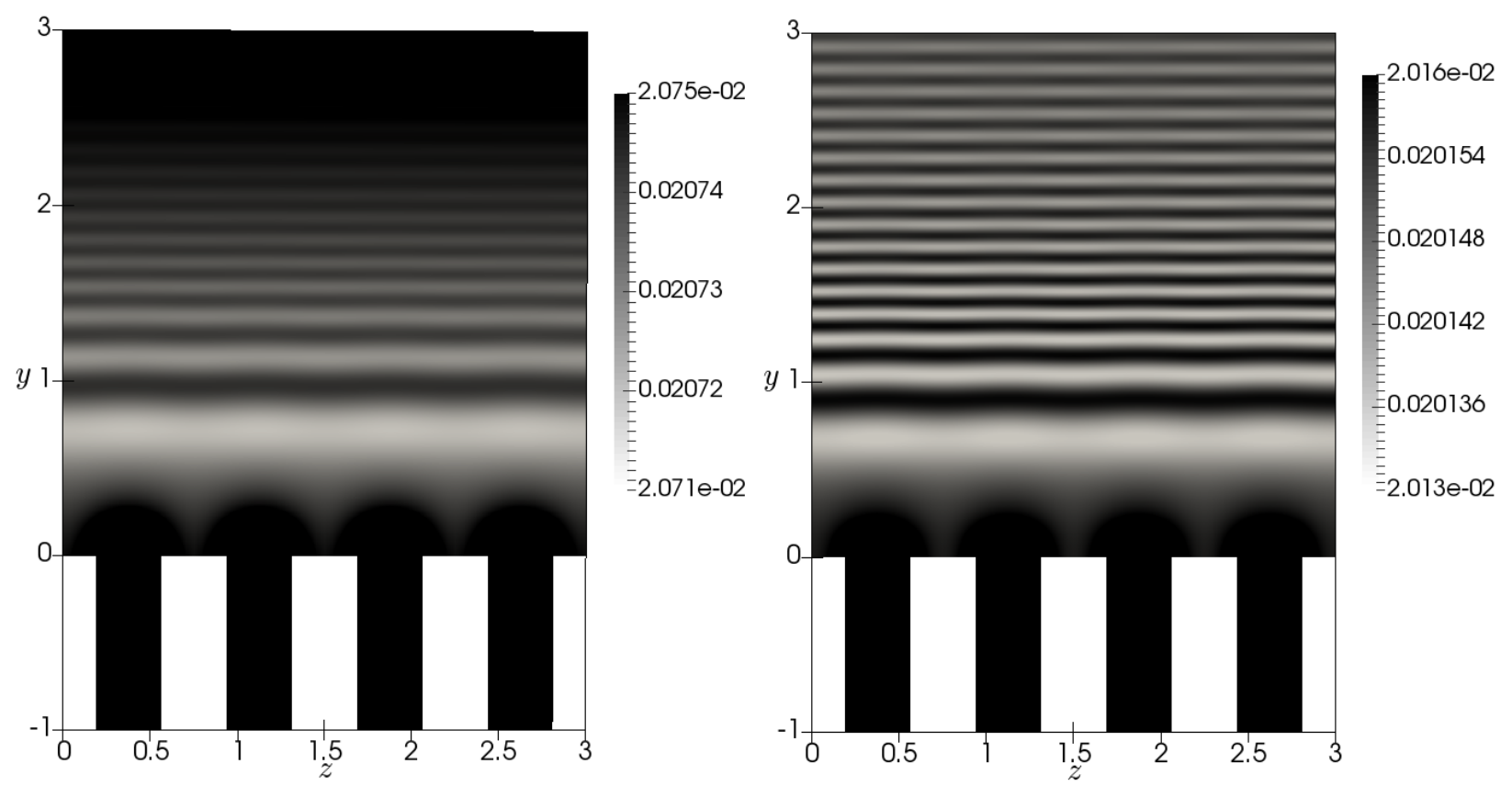

Figure 9: Pressure field on the $z y$-plane at $x=1.85$ at $\operatorname{Re}=6000$ (left) and $\operatorname{Re}=20000$ (right). Time $t=50$
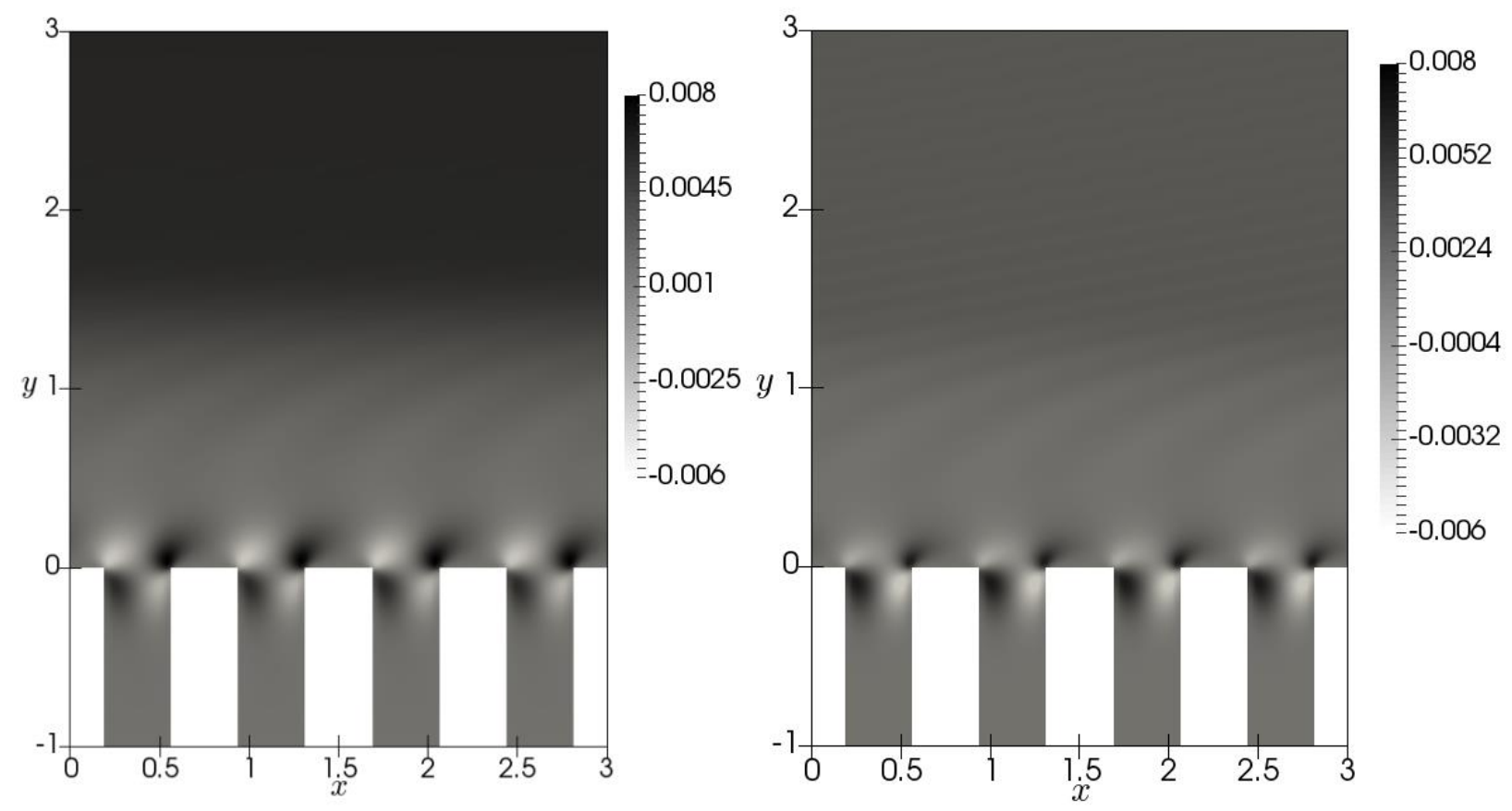

Figure 10: Vertical velocity field on the $x y$-plane at $z=1.85$ at $R e=6000$ (left) and $R e=$ 20000 (right). Time $t=50$ 


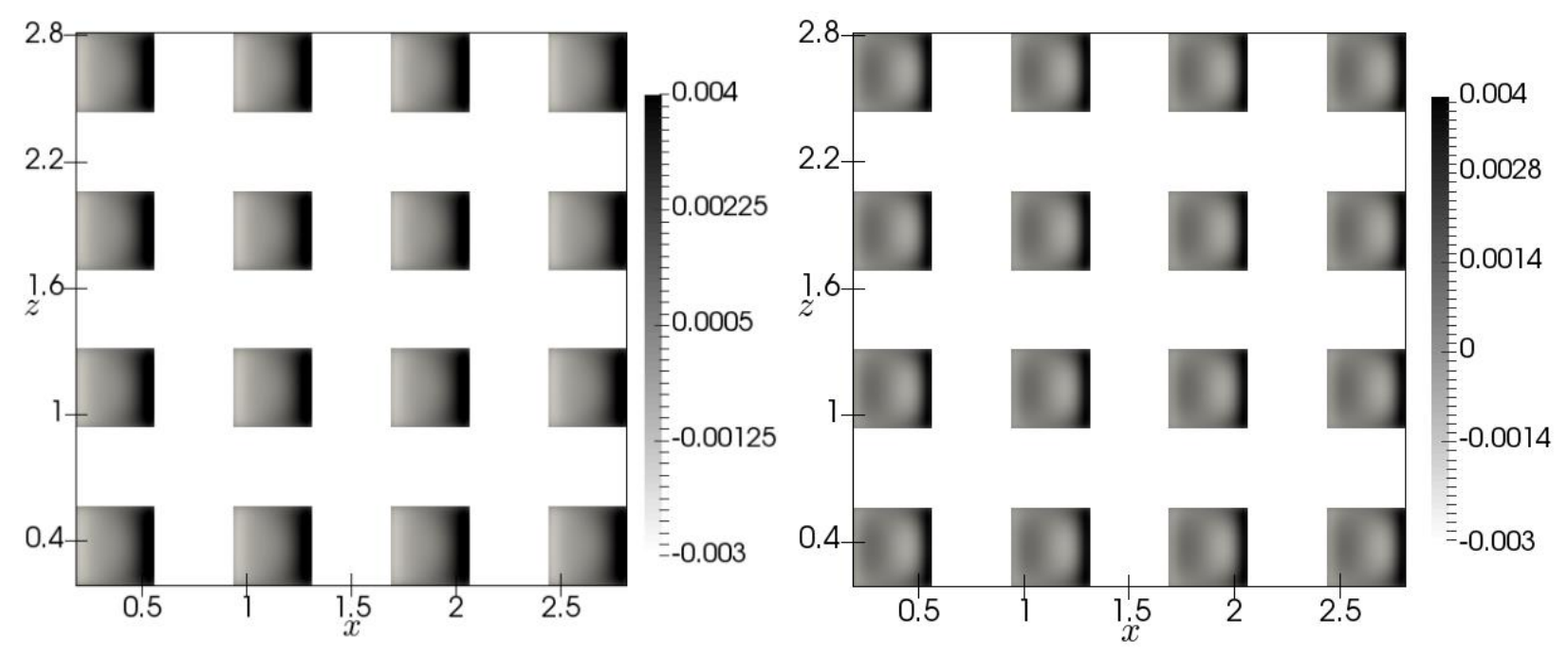

Figure 11: Vertical velocity field on the $x z$-plane at $y=0$ at $R e=6000$ (left) and $R e=20000$ (right). Time $t=50$

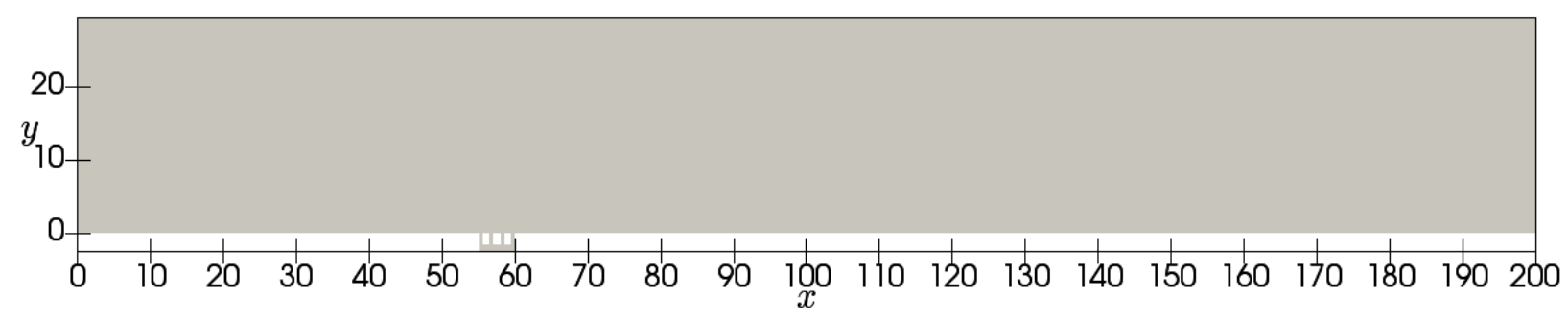

Figure 12: Computational domain, flat plate with slots and plenum chamber located in the region $x=55-60$

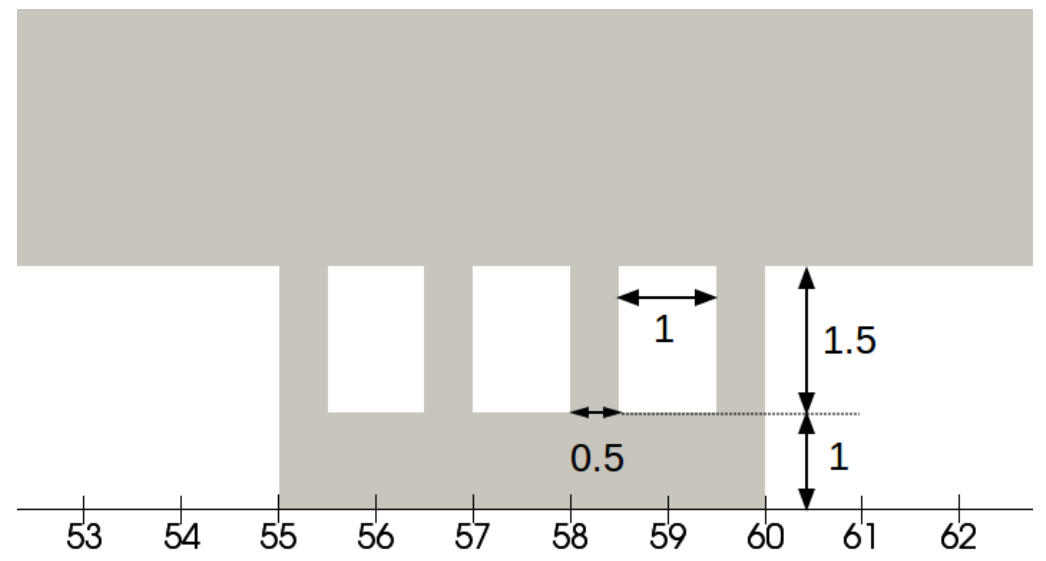

Figure 13: Close-up of the slot region 


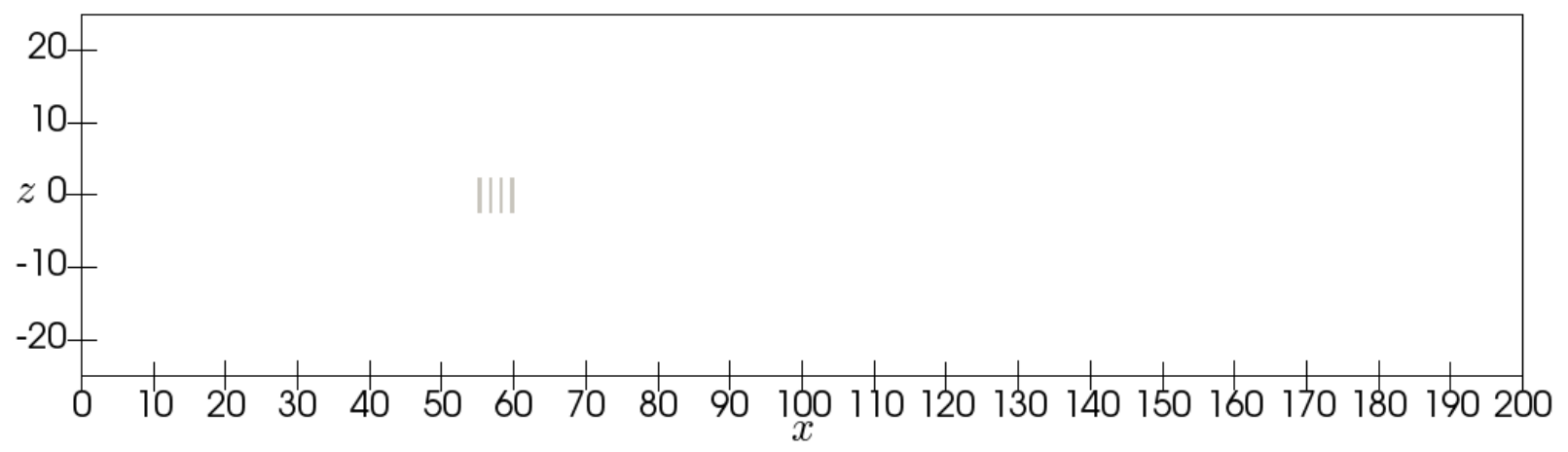

Figure 14: Top view of the domain in the $x z$-plane at $y=0$
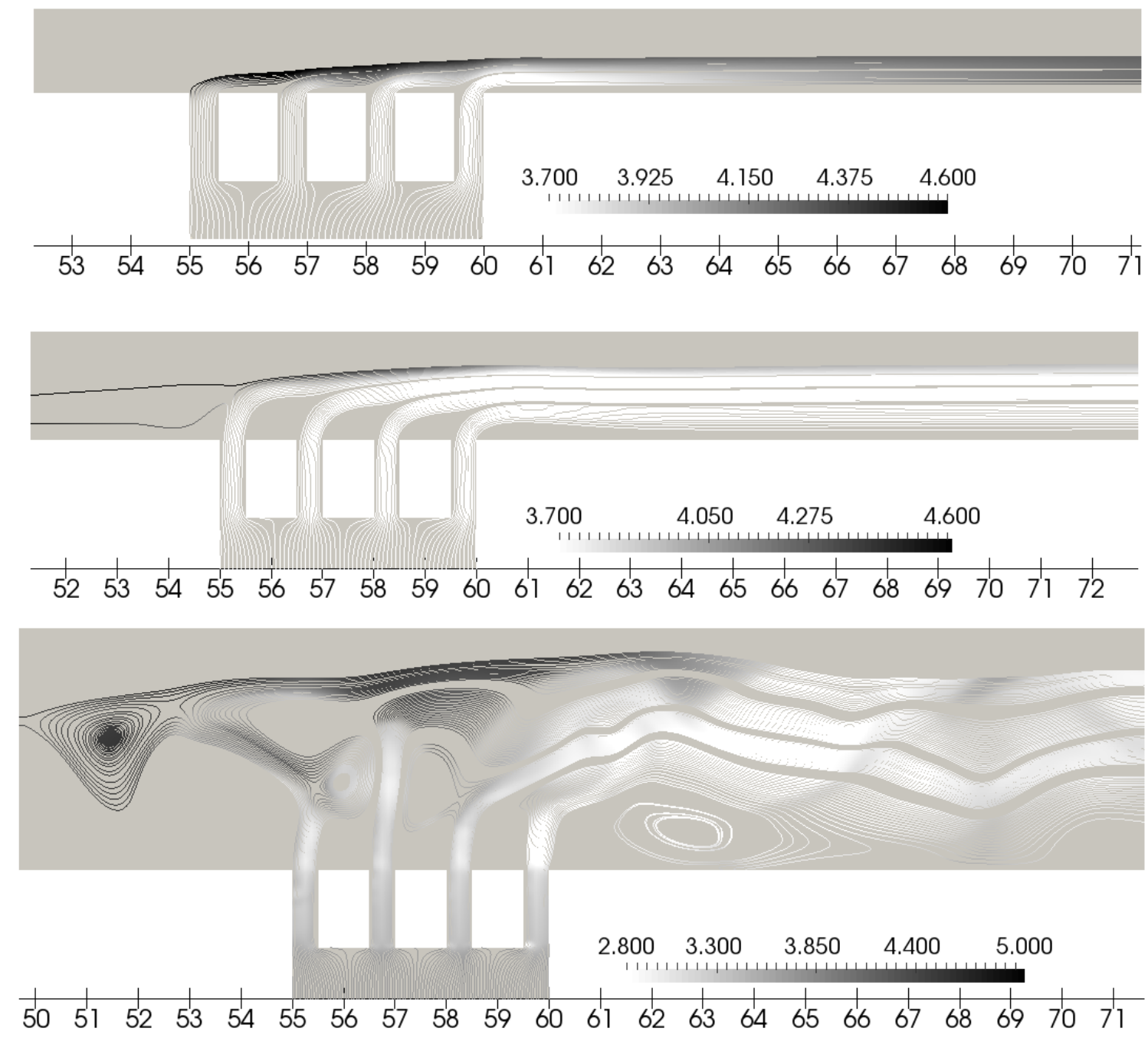

Figure 15: Temperature streamlines in the injection region $-p_{0}=1.2 p_{\infty}$ (top), $p_{0}=1.5 p_{\infty}$ (middle), $p_{0}=3 p_{\infty}($ bottom $)$ 

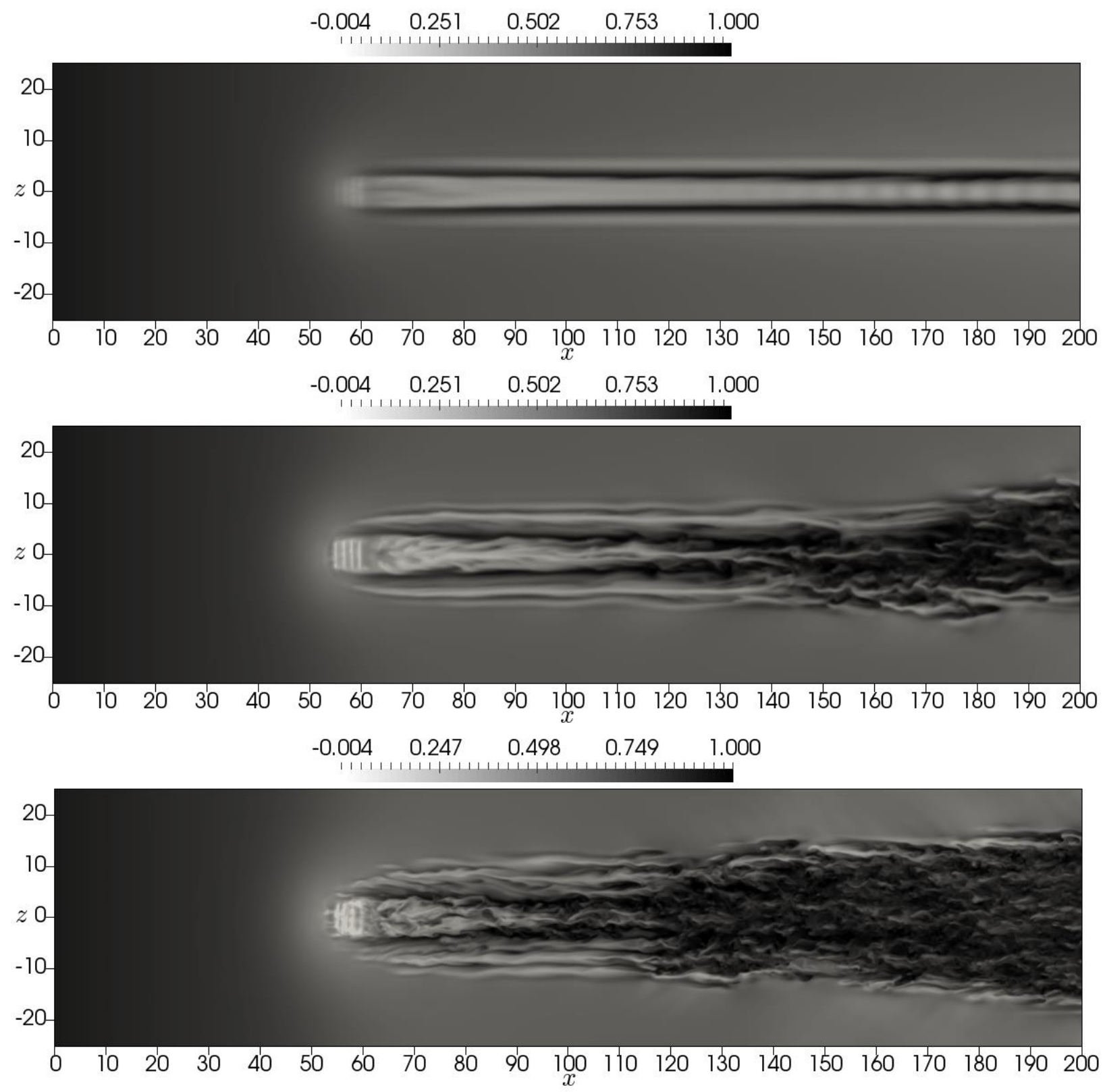

Figure 16: Streamwise velocity $(u)$ inside the boundary layer at the height $y=1-p_{0}=1.5 p_{\infty}$ (top), $p_{0}=2 p_{\infty}$ (middle), $p_{0}=3 p_{\infty}$ (bottom) 
$\begin{array}{lllll}-0.200 & 0.15 & 0.5 & 0.85 & 1.200\end{array}$

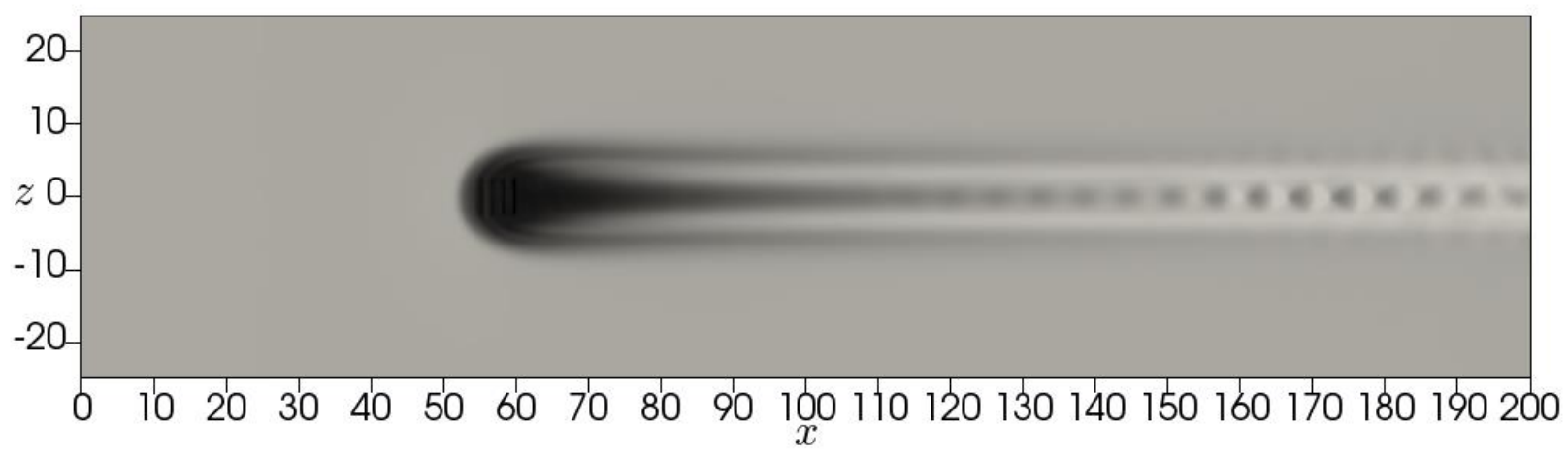
$\begin{array}{lllll}-0.200 & 0 & 0.35 & 0.7 & 1.200\end{array}$

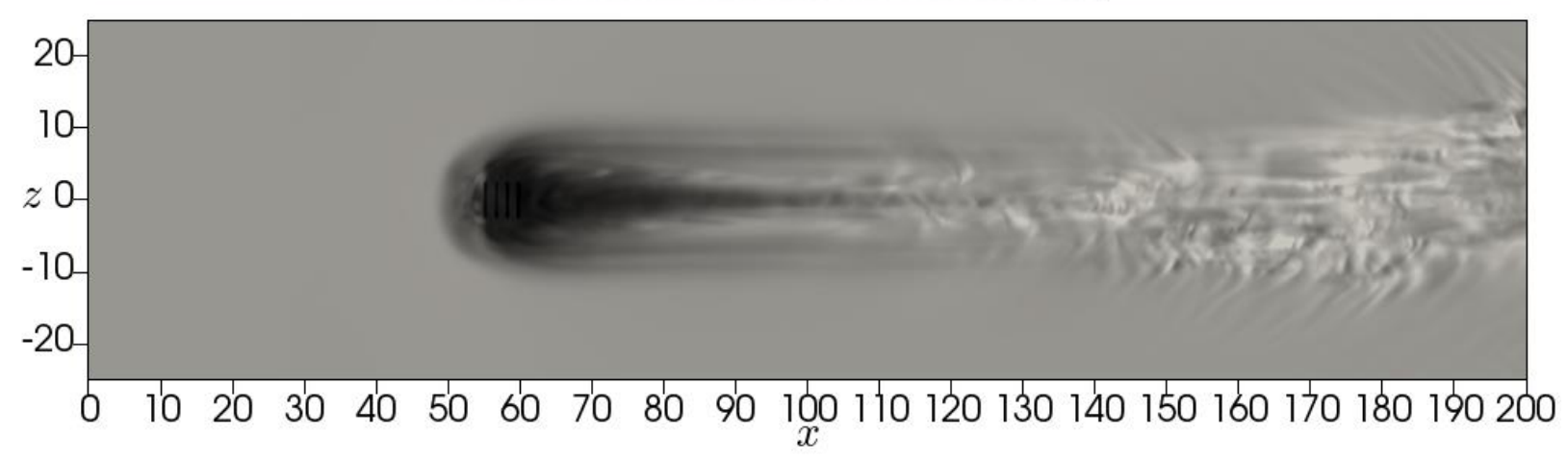

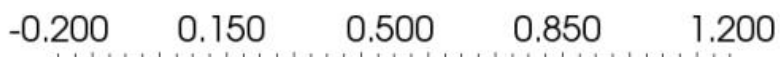

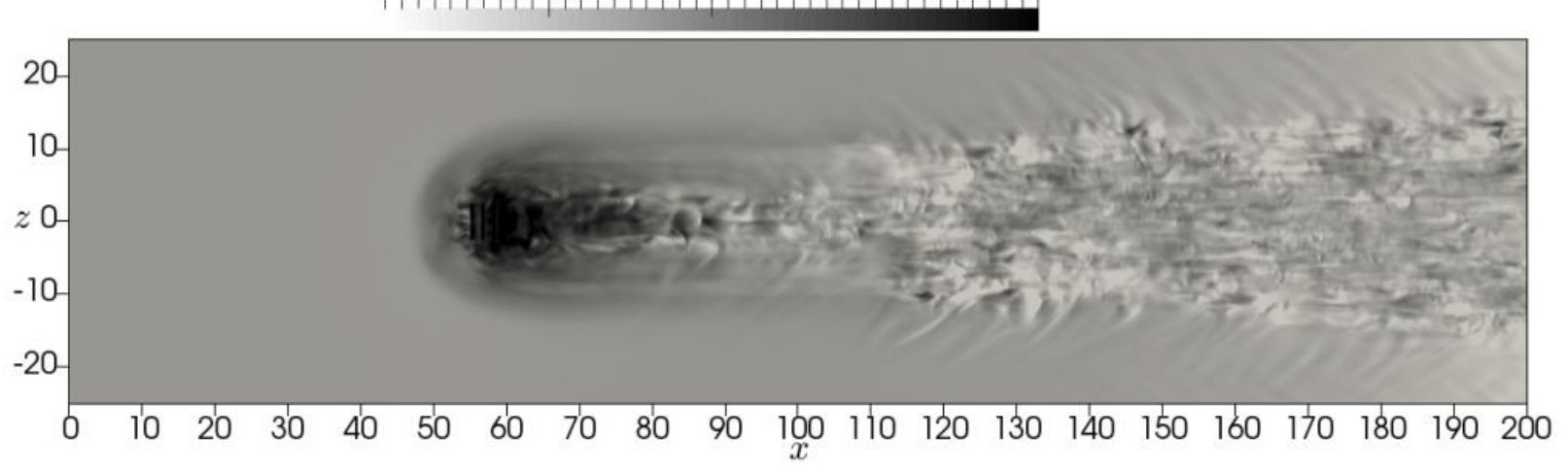

Figure 17: Cooling effectiveness on the surface $-p_{0}=1.5 p_{\infty}$ (top), $p_{0}=2 p_{\infty}$ (middle), $p_{0}=3 p_{\infty}$ (bottom) 


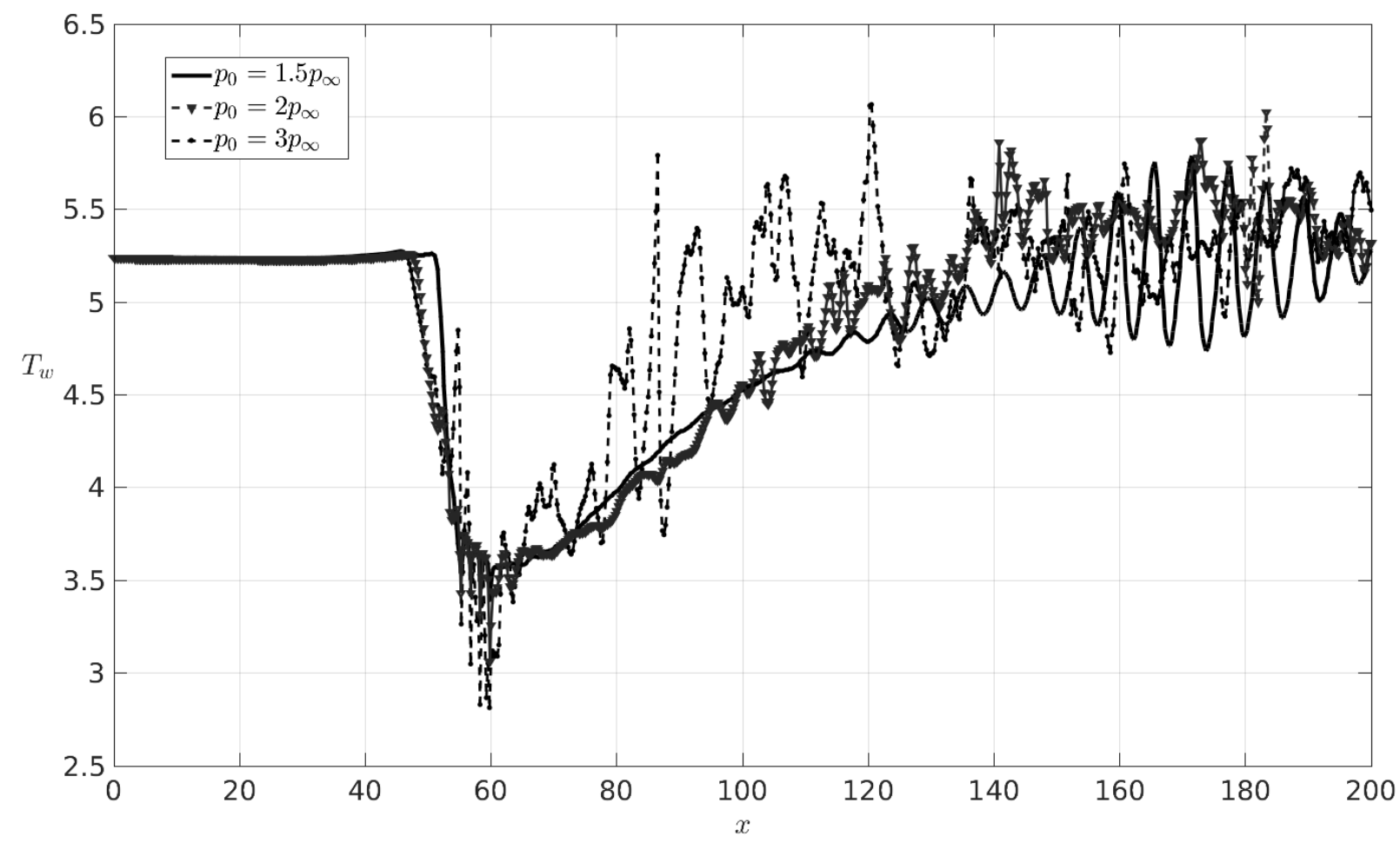

Figure 18: Surface temperature along the midspan axis $(z=0)$

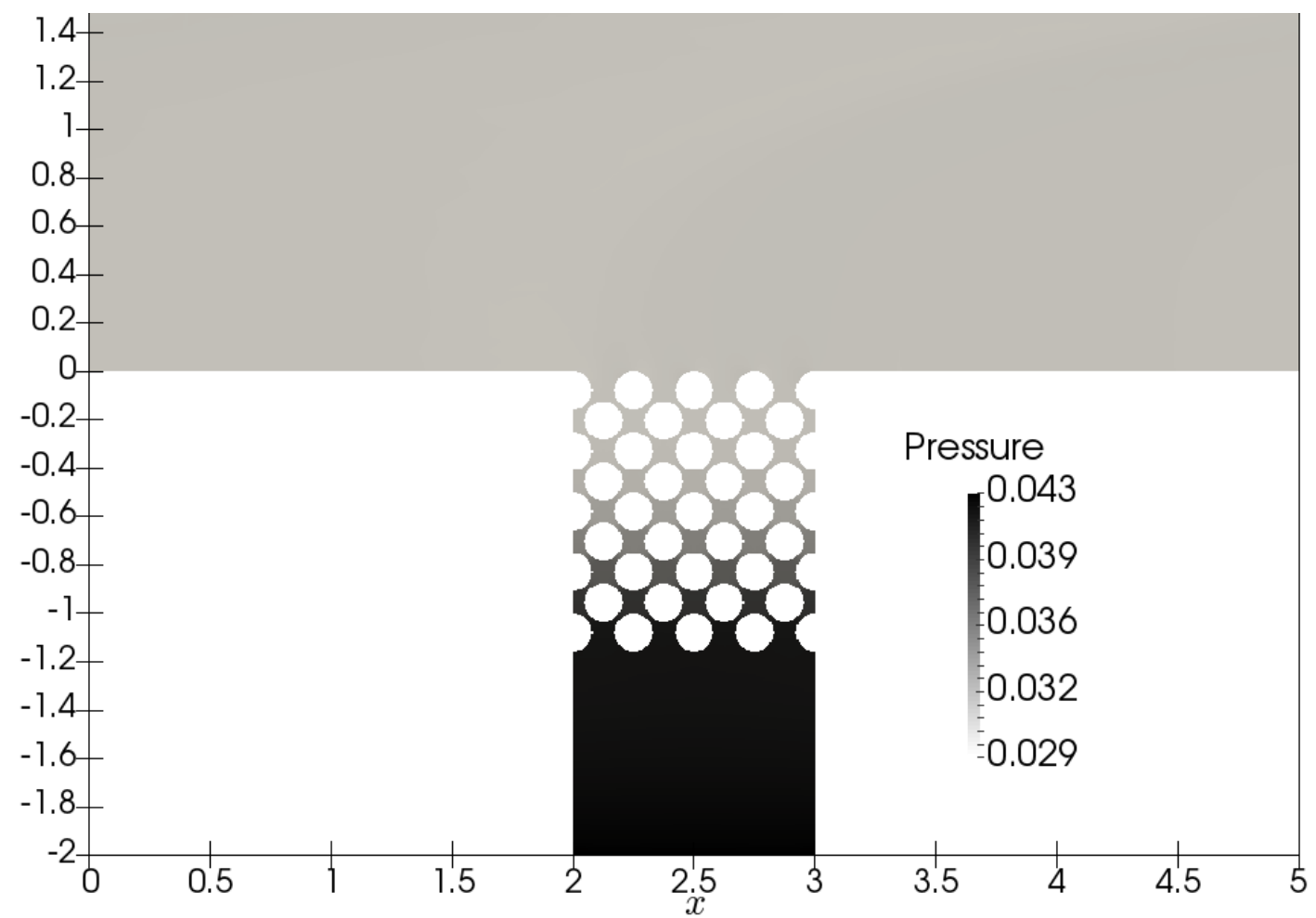

Figure 19: Pressure field 

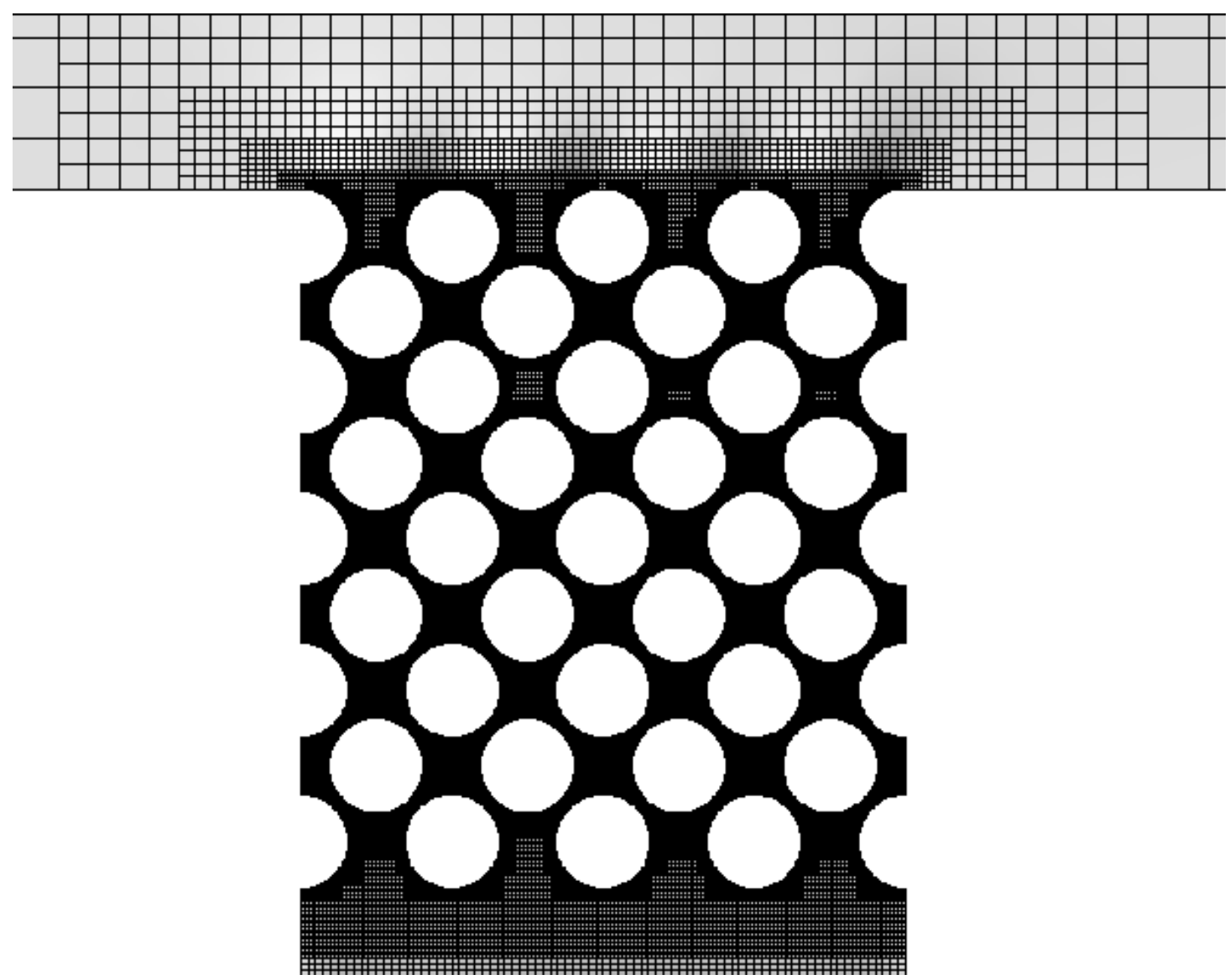

Figure 20: Grid with 6 AMR levels. Momentum in the $y$-direction plotted

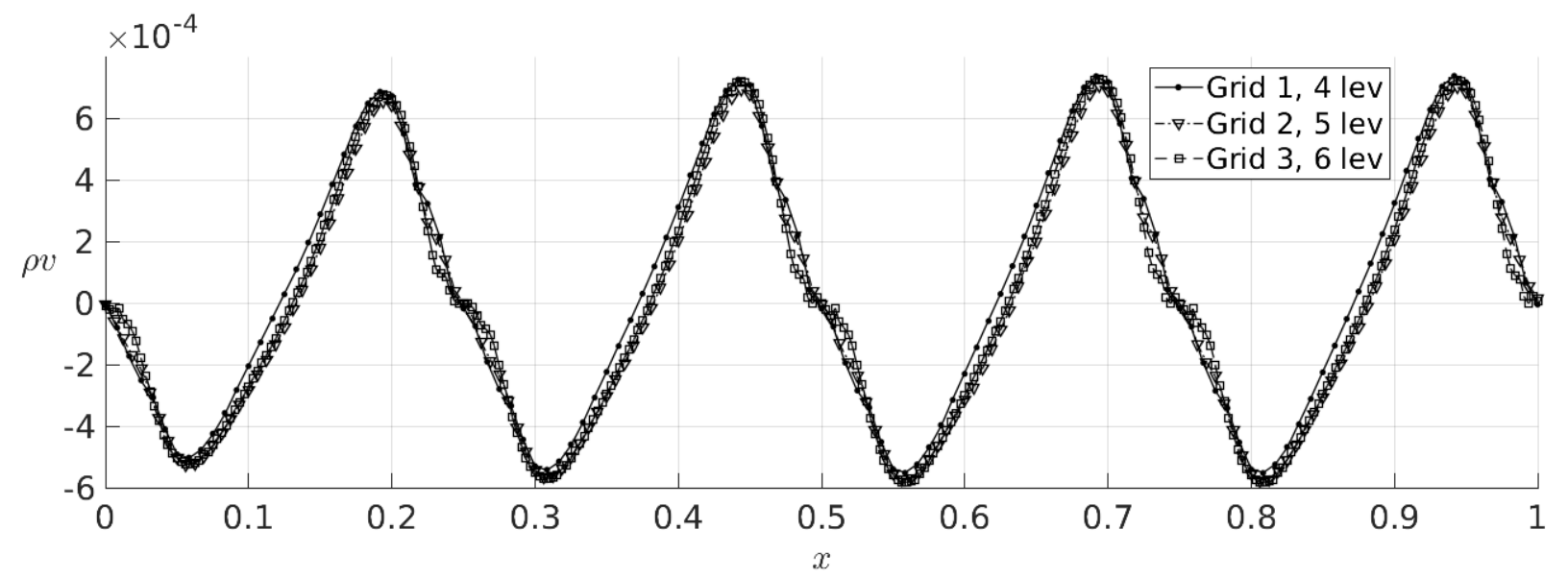

Figure 21: Blowing ratio along the $x$-direction on the surface - grid convergence study 


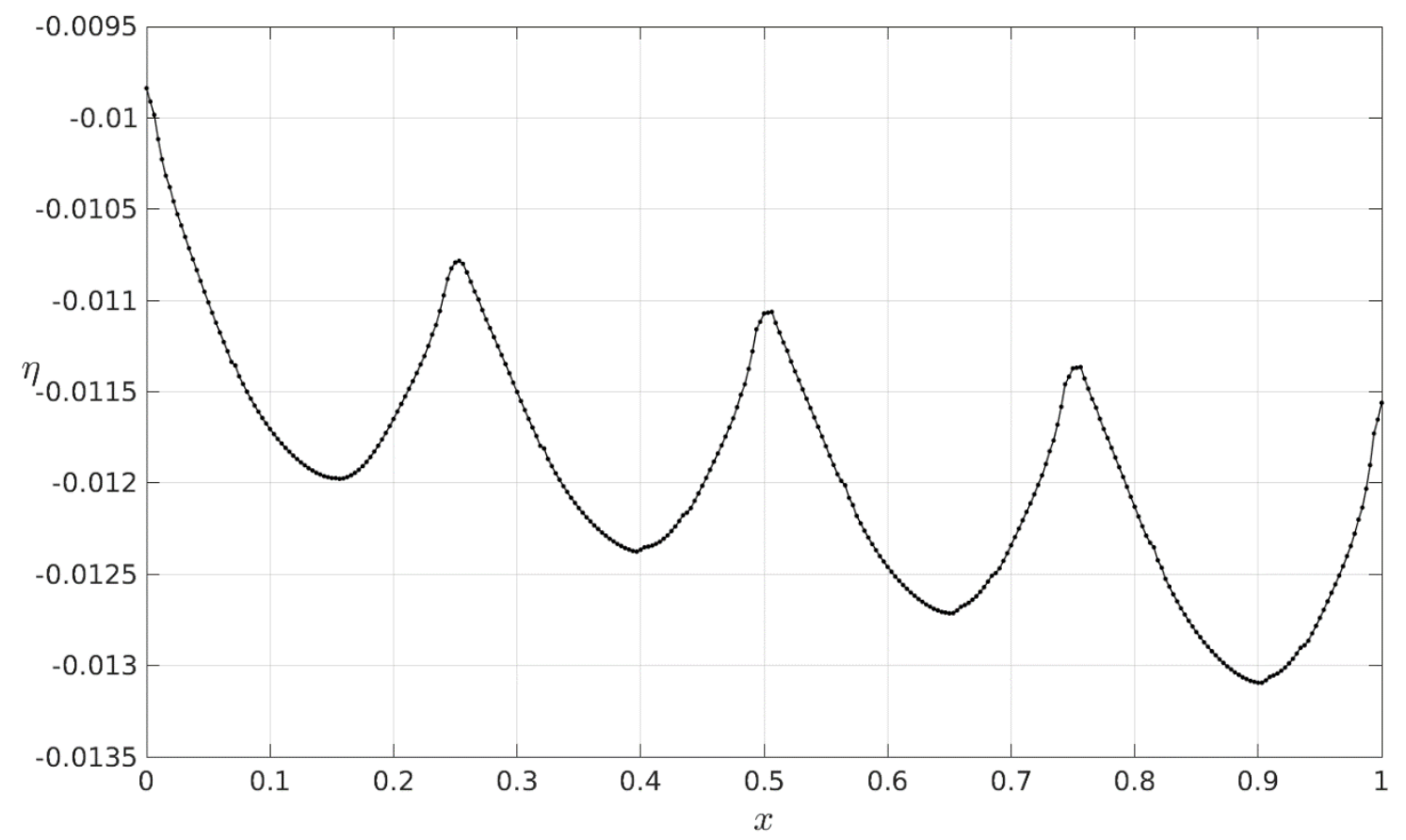

Figure 22: Cooling effectiveness along the $x$-direction on the surface

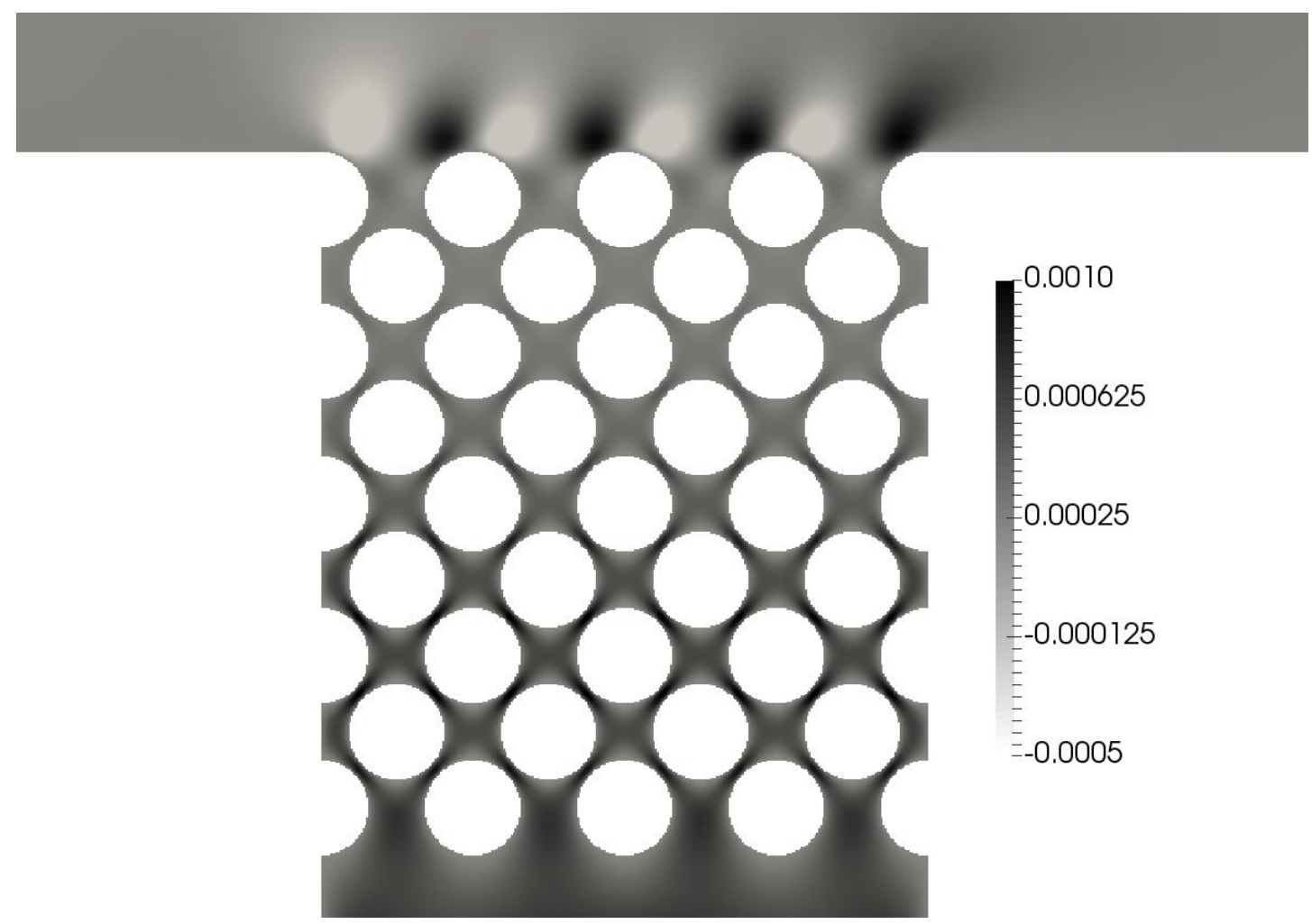

Figure 23: Field of the momentum in the $y$-direction $(\rho v)$. Close-up in the porous layer 

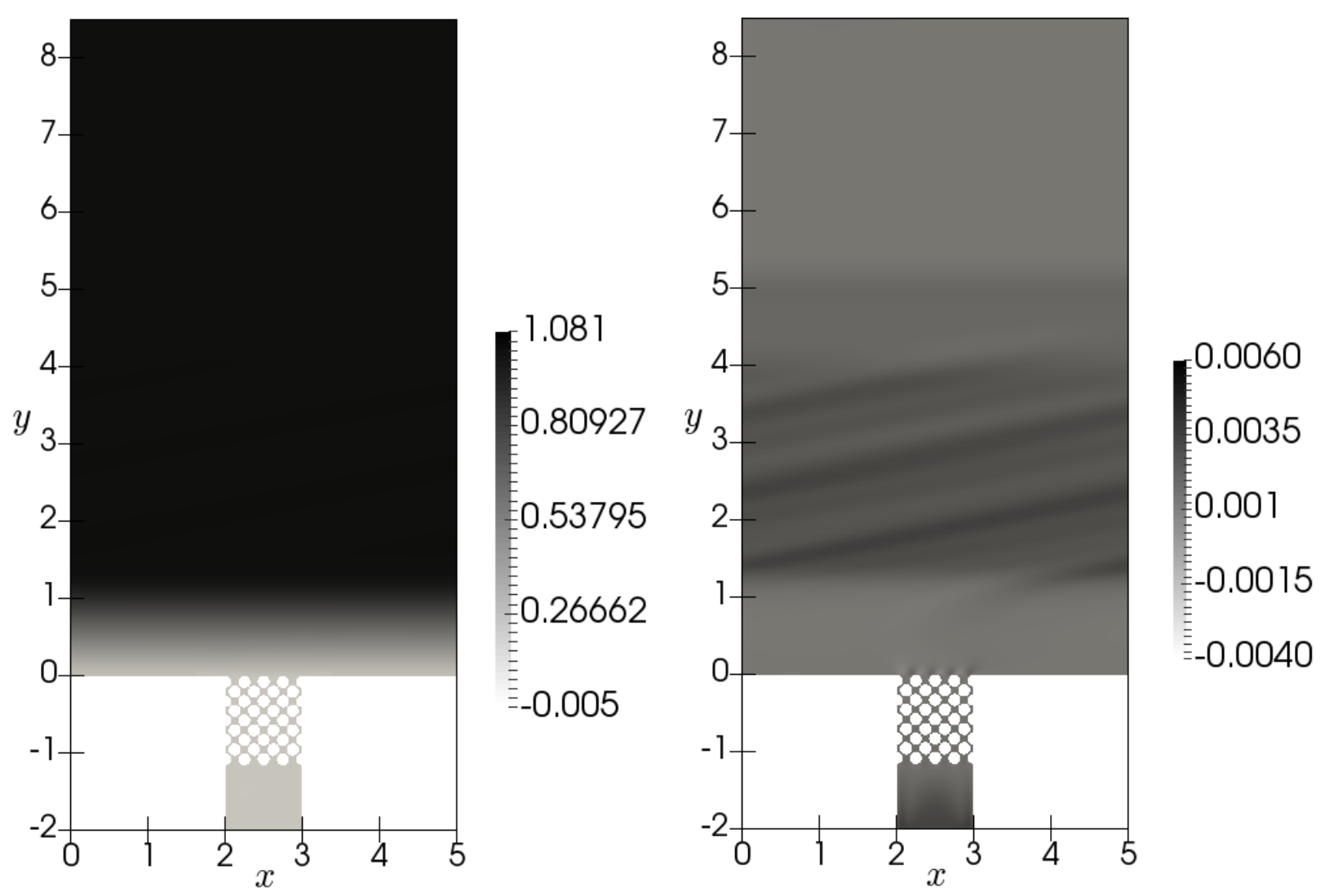

Figure 24: Streamwise (left) and vertical (right) velocity fields. Time $t=20$ 